\title{
Sliding Mode Control of Cable-Driven Redundancy Parallel Robot with 6 DOF Based on Cable-Length Sensor Feedback
}

\author{
Wei Lv, Limin Tao, and Zhengnan Ji \\ Science and Technology on Integrated Logistics Support Laboratory, National University of Defense Technology, \\ Changsha 410000, China \\ Correspondence should be addressed to Wei Lv; lvwei1987@outlook.com
}

Received 9 February 2017; Accepted 23 April 2017; Published 23 May 2017

Academic Editor: Oscar Reinoso

Copyright (C) 2017 Wei Lv et al. This is an open access article distributed under the Creative Commons Attribution License, which permits unrestricted use, distribution, and reproduction in any medium, provided the original work is properly cited.

\begin{abstract}
The sliding mode control of the cable-driven redundancy parallel robot with six degrees of freedom is studied based on the cablelength sensor feedback. Under the control scheme of task space coordinates, the cable length obtained by the cable-length sensor is used to solve the forward kinematics of the cable-driven redundancy parallel robot in real-time, which is treated as the feedback for the control system. First, the method of forward kinematics of the cable-driven redundancy parallel robot is proposed based on the tetrahedron method and Levenberg-Marquardt method. Then, an iterative initial value estimation method for the LevenbergMarquardt method is proposed. Second, the sliding mode control method based on the exponential approach law is used to control the effector of the robot, and the influence of the sliding mode parameters on control performance is simulated. Finally, a six-degreeof-freedom position tracking experiment is carried out on the principle prototype of the cable-driven redundancy parallel robot. The experimental results show that the robot can accurately track the desired position in six directions, which indicates that the control method based on the cable-length sensor feedback for the cable-driven redundancy parallel robot is effective and feasible.
\end{abstract}

\section{Introduction}

A multi-DOF heave compensation crane can compensate for the relative movement of two ships during a replenishment operation at sea, as shown in Figure 1. The cable-driven redundancy parallel robot (CDRPR) is an important part of the heave compensation crane and can compensate for the six-degree-of-freedom (6-DOF) motion of a wave-induced ship during the replenishment operation. The CDRPR is a type of cable-driven parallel mechanism whereby the cable number is more than the freedom; it can lift large cargo, such as containers, using cables. The robot offers the advantages of a large working space, small inertia, and strong antiswing ability [1].

There are two control schemes for CDRPR based on the coordinates used [2], namely, the task and joint space coordinate. The control system controls the length of the cable obtained by sensors to reach the ideal value in the joint space coordinates, which controls the position of the end-effector [3-5]. The end-effector pose obtained from a 6-DOF position sensor or other methods is treated as a feedback for the control system in task space coordinates [6-8]. The control scheme of the task space coordinates is more accurate than other approaches for three reasons. First, in the joint space control case, the cable coupled behavior and its total effect on the end-effector position are ignored [9]. Second, in the task space coordinates, the end-effector position error input to the control system is the one between the current value and ideal value. Third, in the joint space coordinates, the end-effector pose is changed without changing the joint control parameter if there is a jam in the system. However, the task control can observe the change [10].

Some sensors should nevertheless be used to obtain the end-effector pose rapidly and accurately, and this is then treated as feedback for the control system in the task space control. There are many sensors to measure the pose of the end-effector, namely, the computer vision sensor, laser sensor, and cable-length sensor. The computer vision sensor is a wellknown solution that makes it possible for the parallel robot to acquire the pose of the end-effector. Bayani et al. [11] introduced a vision-based position control for a planar cabledriven robot. Dallej et al. $[12,13]$ proposed a vision-based 


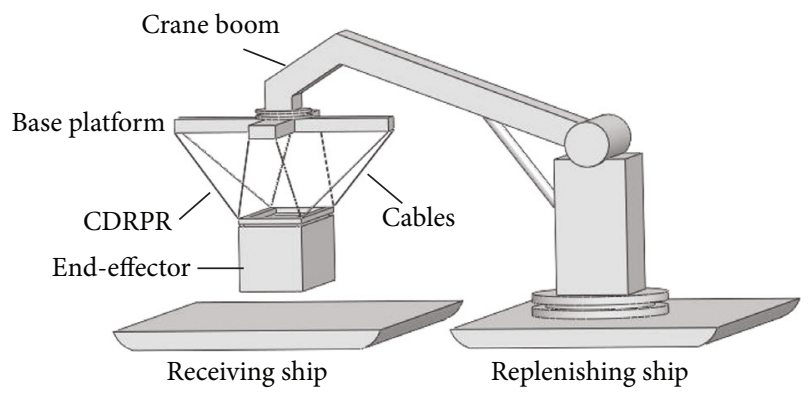

FIGURE 1: Replenishment operation at sea.

control method for ReelAx8 using a 3D pose visual serving sensor. Lu et al. [14] controlled the large radio telescope robot with a feedback system that is based on a combination of the vision sensor and encoder. Ramadour et al. [15] solved the translational velocity sent to the controller using a vision sensor that was installed on the platform of Marionet-Assist. Chellal et al. [16] introduced an INCA 6D robot that was equipped with a vision sensor Bonita used to measure the pose of the end-effector. However, the computer vision sensor is easily affected by weather such as rain, fog, and nighttime darkness. Measurement accuracy is reduced in the wave replenishment operation. The laser sensor can obtain an accurate and rapid position measure. Newman et al. [17] calibrated the Motoman P8 robot using a laser tracking sensor. In addition, the orientation measure is not mastered. It is only used for kinematic identification and has never been used in the control loop. Lytle et al. [18] used a laser-based 3D sensor to track RoboCrane's pose. However, it is expensive. The use of other sensor measurements [19-22] suffers from disadvantages such as high cost, the complexity of the measurement system, and unavoidable round-off errors and measurement noise at sea.

Another method to obtain the end-effector pose is use of the cable-length sensor. That is, the length of the hoisting cable is measured by a cable-length sensor, and then the end-effector pose is solved based on the forward kinematics (FK) of the CDRPR. However, solving the FK problem of the CDRPR in real-time is a complex undertaking because of the high-dimensional multivariate equations. Gallina et al. [23] and Khosravi and Taghirad [24] used the forward kinematics as the feedback to control planar cable-driven robots. However, the robots are planar robots and not applicable for CDRPR. Many researchers have attempted to solve the FK problem. Williams et al. [25] introduced a full Cartesian pose metrology system using 7-cable robots with a 6-string pot metrology. Yang et al. [26] put forward an FK method named modified global Newton-Raphson. Sun-an and Yamin [27] proposed a mixed algorithm combining immune evolutionary algorithm and numerical iterative. Morell et al. $[28,29]$ and Tarokh [30] proposed a method to solve the FK problem. Unlike other approaches, the algorithm does not use geometric parameters. He et al. [31] proposed multitask Gaussian method to solve FK problem for 6-DOF Stewart platform. Lee and Shim $[32,33]$ present an elimination procedure method to solve the FK problem, whereas $\mathrm{Xu}$ and
Xi [34] present a real-time method for a tripod FK. Pott [35] proposes a method to solve the FK problem of an IPAnema cable robot using interval techniques and an iterative solver. However, most of the above methods are used on a Stewart platform and are inapplicable to CDRPR.

In this paper, a feedback method for sliding mode control based on the cable-length sensor is proposed to control the CDRPR. Compared with other methods, the feedback method of the cable-length sensor offers the advantages of low cost, no environmental impact, no measurement noise, high speed, high precision, and suitability for marine environments, which is a suitable feedback method for task space control for CDRPR. To solve the problem of high-dimensional multivariate equations, the FK problem of the CDRPR is transformed into the tetrahedron approach and optimum theory. In this paper, the high-dimensional nonlinear equations of mutual coupling were transformed into independent low-dimensional nonlinear equations using the tetrahedron approach (TA). In this manner, the lowdimensional nonlinear equations could be calculated using the Levenberg-Marquardt (LM) method. Then, the forward kinematics could be solved in real-time.

This article contains many sections. Section 1 introduces the basic aspects of the CDRPR, including a literature review and the basis of this article. Section 2 describes the structural design and the coordinates of the CDRPR in addition to the FK of the CDRPR. Section 3 introduces the dynamic of the CDRPR. Section 4 discusses the problem of sliding mode control based on exponential approach law. Section 5 shows the experimental studies on simulations and control of the principal prototype. Section 6 discusses the conclusions and problems for further work.

\section{Forward Kinematics}

2.1. System Description. As shown in Figure 2, the CDRPR is composed of the base platform, end-effector, servo systems, cable-length sensor, cable-tension sensor, cables, and corresponding mechanical system. The end-effector is driven by the servo systems. The cable-length sensor measures the length of the cable. The cable-tension sensor measures the tension of the cable. Every two adjacent ropes are attached to a corner of the end-effector, and those ropes attached to the adjacent corner of the end-effector are located close to each other. The inertial frame $\mathcal{N}$ is placed in the inertial space. Frames $\mathscr{B}$ and $\mathscr{P}$ are placed at the base platform and the endeffector, respectively. The origin of frame $\mathscr{B}$ lies in the centre of mass of the base platform, and the origin of frame $\mathscr{P}$ is located at the centre of the upper surface of the end-effector. Let $\mathbf{L}_{i} \in R^{3}(i=1,2, \ldots, 8)$ be the vector of the $i$ th cable. $L_{i}$ is the cable length. $e_{i}$ is the unit vector of $\mathbf{L}_{i} . B_{i}(i=1, \ldots, 8)$ and $P_{j}(j=1, \ldots, 4)$ are the attachment points. $\mathbf{p}_{j}$ is the vector from $O_{P}$ to $P_{j}$ expressed in frame $\mathscr{P}$, and $\mathbf{B}_{i}$ is the vector from $\mathrm{O}_{B}$ to $B_{i}$.

2.2. Inverse Kinematics. According to geometry relation shown in Figure 2, $\mathbf{L}_{i}$ is

$$
\mathbf{L}_{i}=\mathbf{B}_{i}-\left(\mathbf{x}_{P B}+\mathbf{R}_{P} \mathbf{p}_{j}\right),
$$




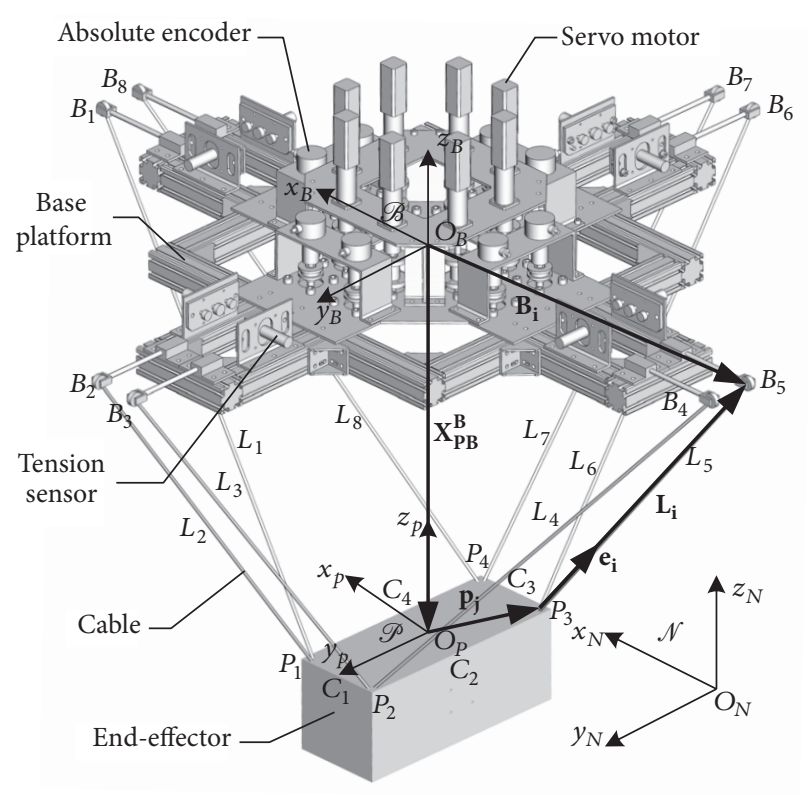

Figure 2: Structure of the CDRPR.

where $\mathbf{x}_{P B}$ is the position vector from the end-effector to the base platform. $\mathbf{R}_{P}$ is the rotation matrix from the end-effector to the base platform. If $\mathbf{x}_{P B}$ and $\mathbf{R}_{P}$ are obtained, then the length of the $i$ th cable is

$$
\begin{aligned}
& L_{i}=\left|\mathbf{L}_{i}\right|=\sqrt{\left(\mathbf{B}_{i}-\mathbf{x}_{P B}-\mathbf{R}_{P} \mathbf{p}_{j}\right)^{T}\left(\mathbf{B}_{i}-\mathbf{x}_{P B}-\mathbf{R}_{P} \mathbf{p}_{j}\right)} \\
& (j=1, i=1,2 ; j=2, i=3,4 ; j=3, i=5,6 ; j=4, i=7,8) .
\end{aligned}
$$

The constraint equations of FK problem exported by (2) are

$$
\begin{aligned}
& f_{i}^{\prime}\left(x^{\prime}, y^{\prime}, z^{\prime}, \phi^{\prime}, \theta^{\prime}, \psi^{\prime}\right) \\
& \quad=\left(\mathbf{B}_{i}-\mathbf{x}_{P}-\mathbf{R}_{P} \mathbf{p}_{i}\right)^{T}\left(\mathbf{B}_{i}-\mathbf{x}_{P}-\mathbf{R}_{P} \mathbf{p}_{i}\right)-L_{i}^{2}=0 \\
& (i=1, \ldots, 8),
\end{aligned}
$$

where $x^{\prime}, y^{\prime}, z^{\prime}, \phi^{\prime}, \theta^{\prime}, \psi^{\prime}$ are the position and pose variables, respectively, of the end-effector relative to the base platform.

The CDRPR is a redundant cable-driven parallel robot. As is shown in (3), different from the traditional rigid parallel robot, the CDRPR has eight cables, and the FK solution is a function of the eight variables. The process of computation is in a six-dimensional space. It is difficult to provide a unique FK solution in real-time for the CDRPR since (3) is expressed as high-dimensional coupling equation. If the numerical iterative algorithm is used to calculate (3) directly, the six-dimensional matrix and the corresponding inverse matrix will be calculated at each step. It will increase the amount of calculation. It will take a long time to solve the FK problem and it is not possible to feed back the end-effector poses to the control system in real-time.

In this paper, four real variables are used instead of the six variables of (3). The eight-dimensional coupling equation is translated into the four-dimensional decoupling equation, which reduces the amount of iterative computation. Then, the LM could converge rapidly to the convergence point. The end-effector poses could be returned to the control system immediately since the FK would be solved in real-time.

2.3. Forward Kinematics Based on TA. The vertices of the end-effector are treated as the top vertex of the tetrahedron. The space composed of eight ropes is divided into four tetrahedrons based on the tetrahedron lemma [36]. The plane of the base platform is the base of the tetrahedron. The two lines that attach the same vertices of the end-effector are the space lines of the tetrahedron. The line that attaches point $O_{B}$ and the end-effector vertex is the third space line, $\lambda_{i}$, as shown in Figure 3.

Let the vector $\mathbf{X}, \mathbf{Y}, \mathbf{Z}$ be the coordinate of tetrahedron, and let $\mathbf{X}=\mathbf{B}_{1} /\left\|\mathbf{B}_{1}\right\|, \mathbf{Y}=\left(-\left(\mathbf{B}_{2} \cdot \mathbf{X}\right) \mathbf{X}+\mathbf{B}_{2}\right) /\left\|-\left(\mathbf{B}_{2} \cdot \mathbf{X}\right) \mathbf{X}+\mathbf{B}_{2}\right\|$, and $\mathbf{Z}=\mathbf{X} \times \mathbf{Y}, \mathbf{B}_{i}=\overrightarrow{O_{B} B_{i}}$. The first tetrahedron includes two vertices $\left(P_{1}, \mathbf{B}_{1}, \mathbf{B}_{2}, O_{B}\right) . P_{1}$ is the top vertex, and $\lambda_{1}$ is unknown, where $\lambda_{1}=\left\|\mathbf{P}_{\mathbf{1}}\right\|$.

$$
\begin{aligned}
& \mathbf{P}_{1}=\lambda_{1}, \\
& \lambda_{1}=\lambda_{11} \mathbf{X}+\lambda_{12} \mathbf{Y}-\lambda_{13} \mathbf{Z},
\end{aligned}
$$

where $\lambda_{11}=\left(\lambda_{1}^{2}+B_{1}^{2}-L_{1}^{2}\right) / 2\left\|\mathbf{B}_{1}\right\|, \lambda_{12}=\left(\lambda_{1}^{2}+B_{2}^{2}-L_{2}^{2}-\right.$ $\left.2 \lambda_{11}\left(\mathbf{B}_{2} \cdot \mathbf{X}\right)\right) / 2\left(\mathbf{B}_{2} \cdot \mathbf{Y}\right)$, and $\lambda_{13}^{2}=\lambda_{1}^{2}-\lambda_{11}^{2}-\lambda_{12}^{2}, \mathbf{P}_{i}=\overrightarrow{O_{B} P_{i}}$.

The second tetrahedron includes two vertices $\left(P_{2}, \mathbf{B}_{3}, \mathbf{B}_{4}, O_{B}\right)$, where $P_{2}$ is the top vertex. $\lambda_{2}$ is unknown, where $\lambda_{2}=\left\|\mathbf{P}_{2}\right\|$.

$$
\begin{aligned}
& \mathbf{P}_{2}=\lambda_{2}, \\
& \lambda_{2}=\lambda_{21} \mathbf{Y}-\lambda_{22} \mathbf{X}-\lambda_{23} \mathbf{Z},
\end{aligned}
$$

where $\lambda_{21}=\left(\lambda_{2}^{2}+B_{3}^{2}-L_{3}^{2}\right) / 2\left\|\mathbf{B}_{3}\right\|, \lambda_{22}=\left(\lambda_{2}^{2}+B_{4}^{2}-L_{4}^{2}-\right.$ $\left.2 \lambda_{21}\left(\mathbf{B}_{3} \cdot \mathbf{Y}\right)\right) /-2\left(\mathbf{B}_{4} \cdot \mathbf{X}\right)$, and $\lambda_{23}^{2}=\lambda_{2}^{2}-\lambda_{21}^{2}-\lambda_{22}^{2}$.

The third tetrahedron includes two vertices $\left(P_{3}, \mathbf{B}_{5}, \mathbf{B}_{6}\right.$, $\mathrm{O}_{B}$ ), where $P_{3}$ is the top vertex. $\lambda_{3}$ is unknown, where $\lambda_{3}=$ $\left\|\mathbf{P}_{3}\right\|$.

$$
\begin{aligned}
& \mathbf{P}_{3}=\lambda_{3}, \\
& \lambda_{3}=-\lambda_{31} \mathbf{X}-\lambda_{32} \mathbf{Y}-\lambda_{33} \mathbf{Z},
\end{aligned}
$$

where $\lambda_{31}=\left(\lambda_{3}^{2}+B_{5}^{2}-L_{5}^{2}\right) / 2\left\|\mathbf{B}_{5}\right\|, \lambda_{32}=\left(\lambda_{3}^{2}+B_{6}^{2}-L_{6}^{2}+\right.$ $\left.2 \lambda_{31}\left(\mathbf{B}_{6} \cdot \mathbf{X}\right)\right) /-2\left(\mathbf{B}_{6} \cdot \mathbf{Y}\right)$, and $\lambda_{33}^{2}=\lambda_{3}^{2}-\lambda_{31}^{2}-\lambda_{32}^{2}$.

The fourth tetrahedron includes two vertices $\left(P_{4}, \mathbf{B}_{7}, \mathbf{B}_{8}\right.$, $O_{B}$ ), where $P_{4}$ is the top vertex. $\lambda_{4}$ is unknown, where $\lambda_{4}=$ $\left\|\mathbf{P}_{4}\right\|$.

$$
\begin{aligned}
& \mathbf{P}_{4}=\lambda_{4}, \\
& \lambda_{4}=-\lambda_{41} \mathbf{Y}+\lambda_{42} \mathbf{X}-\lambda_{43} \mathbf{Z},
\end{aligned}
$$

where $\lambda_{41}=\left(\lambda_{4}^{2}+B_{7}^{2}-L_{7}^{2}\right) / 2\left\|\mathbf{B}_{7}\right\|, \lambda_{42}=\left(\lambda_{4}^{2}+B_{8}^{2}-L_{8}^{2}-\right.$ $\left.2 \lambda_{41}\left(\mathbf{B}_{8} \cdot \mathbf{Y}\right)\right) / 2\left(\mathbf{B}_{8} \cdot \mathbf{X}\right)$, and $\lambda_{43}^{2}=\lambda_{4}^{2}-\lambda_{41}^{2}-\lambda_{42}^{2}$.

$\mathbf{P}_{i}$ are functions of $\lambda_{i}^{2}(i=1,2,3,4)$. The dot-product signs for (4)-(7) are determined by the angles of adjacent vectors. If the angle is an acute one, the sign is "+"; else, it is "-". $L_{i}$ is measured by the cable-length sensor, and $\mathbf{B}_{i}$ 


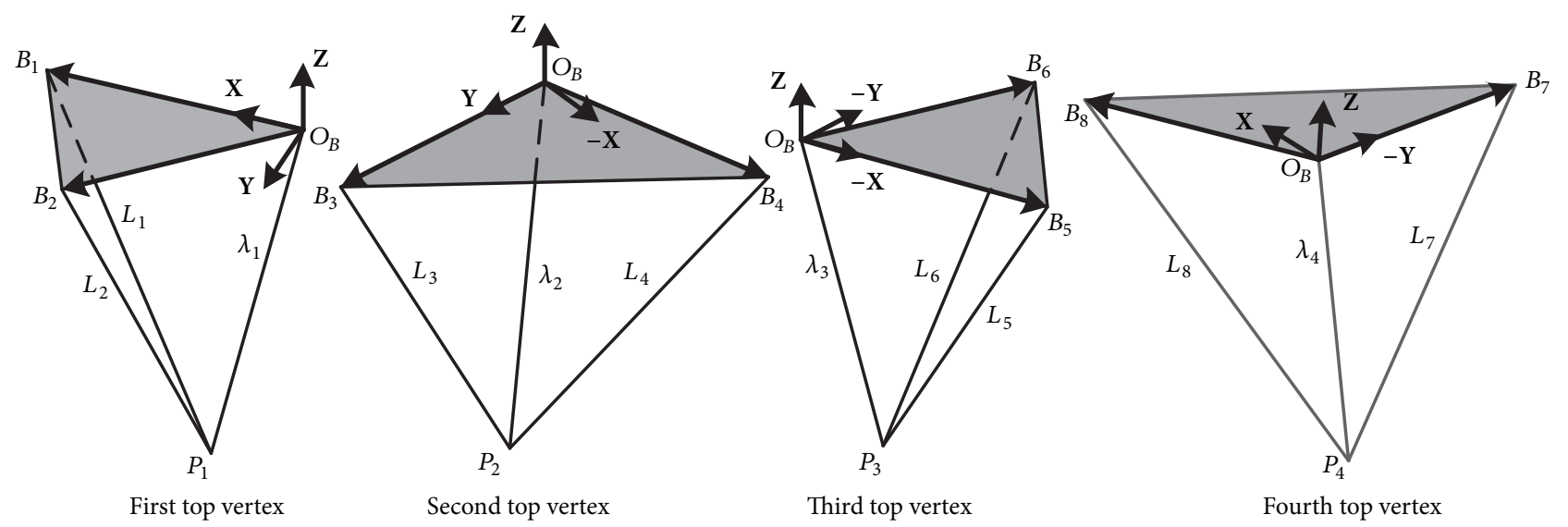

FIGURE 3: Dividing the tetrahedron.

is the structural parameter of the robot. $\lambda_{i}$ is an unknown parameter.

The constraint equations derived by TA can be expressed as follows:

$$
\begin{aligned}
f_{i}\left(\lambda_{i}, \lambda_{j}\right)=\left(\mathbf{P}_{j}-\mathbf{P}_{i}\right)^{T}\left(\mathbf{P}_{j}-\mathbf{P}_{i}\right)-C_{i}^{2}=0, & \\
& (i=1,2,3,4 ; j=2,3,4,1) .
\end{aligned}
$$

That is,

$$
\begin{aligned}
f_{i}\left(\lambda_{i}, \lambda_{j}\right)=G_{1} \lambda_{i}^{2}+G_{2} \lambda_{j}^{2}+G_{3} \lambda_{i}^{2} \lambda_{j}^{2}+G_{4}=0, \\
(i=1,2,3,4 ; j=2,3,4,1),
\end{aligned}
$$

where $G_{i}(i=1,2,3,4)$ is a function of $L_{i}$ and is regarded as constant.

Equation (3) $f_{i}^{\prime}$ is a six-dimensional nonlinear equation group that has eight equations. However, there are four equations in $f_{i}$ and each equation has only two unknown variables. $f_{i}$ is less variable relative to $f_{i}^{\prime}$. There is no coupling between variables and no matrix operation. When $f_{i}$ is solved using the LM method, the objective function and gradient function can be obtained as the analytic solution compared to $f_{i}^{\prime}$. When the appropriate initial conditions are selected, the solution can be achieved by a finite number of iterations because the algorithm does not involve a complicated calculation, such as the inverse matrix operation. In other words, the algorithm has the characteristics of fast convergence and meets the requirement of providing a real-time solution.

The solution of $f_{i}$ is a nonlinear least squares problem. $\lambda_{i}(i=1,2,3,4)$ is solved using the LM method, and the pose is solved using the geometric method. The vector superscript denotes the reference coordinate system, and if the vector is not marked, the reference coordinate system is an inertial coordinate system. The position of the end-effector relative to the base platform $\mathbf{x}_{P}^{B}$ is

$$
\begin{aligned}
\mathbf{x}_{P}^{B} & =[\text { swaying, surging, heaving }]^{T} \\
& =\frac{\mathbf{P}_{1}+\mathbf{P}_{2}+\mathbf{P}_{3}+\mathbf{P}_{4}}{4} .
\end{aligned}
$$

The swaying, surging, and heaving are the displacement of the end-effector in directions $x_{B}, y_{B}$, and $z_{B}$, respectively. The orientation vectors $\mathbf{R}=[\mathbf{u}, \mathbf{v}, \mathbf{w}]$ are

$$
\begin{aligned}
& \mathbf{u}=\frac{\left(\mathbf{P}_{1}-\mathbf{P}_{2}\right) / C_{1}+\left(\mathbf{P}_{4}-\mathbf{P}_{3}\right) / C_{3}}{2}, \\
& \mathbf{v}=\frac{\left(\mathbf{P}_{2}-\mathbf{P}_{3}\right) / C_{2}+\left(\mathbf{P}_{1}-\mathbf{P}_{4}\right) / C_{4}}{2}, \\
& \mathbf{w}=\mathbf{u} \times \mathbf{v},
\end{aligned}
$$

where

$$
\begin{aligned}
& \mathbf{R}=\left[\begin{array}{ccc}
C \phi C \varphi & C \phi S \varphi S \delta-S \phi C \delta & C \phi S \varphi C \delta+S \phi S \delta \\
S \phi C \varphi & S \phi S \varphi S \delta+C \phi C \delta & S \phi S \varphi C \delta-C \phi S \delta \\
-S \varphi & C \varphi S \delta & C \varphi C \delta
\end{array}\right], \\
& C \phi=\cos \phi, \\
& C \varphi=\cos \varphi, \\
& C \delta=\cos \delta \text {, } \\
& S \phi=\sin \phi, \\
& S \varphi=\sin \varphi, \\
& S \delta=\sin \delta \text {, } \\
& \text { pitching }=\phi=\arctan \left(\frac{\mathbf{v}(2)}{\mathbf{w}(1)}\right) \text {, } \\
& \text { rolling }=\theta=\arctan \left(\frac{-\mathbf{u}(3)}{\sqrt{\mathbf{u}(1)^{2}+\mathbf{u}(2)^{2}}}\right) \text {, } \\
& \text { yawing }=\psi=\arctan \left(\frac{\mathbf{u}(2)}{\mathbf{u}(1)}\right) \text {, }
\end{aligned}
$$

where pitching, rolling, and yawing being the angle of rotation around $x_{B}, y_{B}$, and $z_{B}$, respectively. The pose of the end-effector relative to the base platform is $\boldsymbol{\omega}_{P}^{B}=\left[\begin{array}{lll}\phi & \theta & \psi\end{array}\right]^{T}$. Let $\mathbf{X}_{P}^{B}=\left[\mathbf{x}_{P}^{B}, \boldsymbol{\omega}_{P}^{B}\right]^{T}$. 


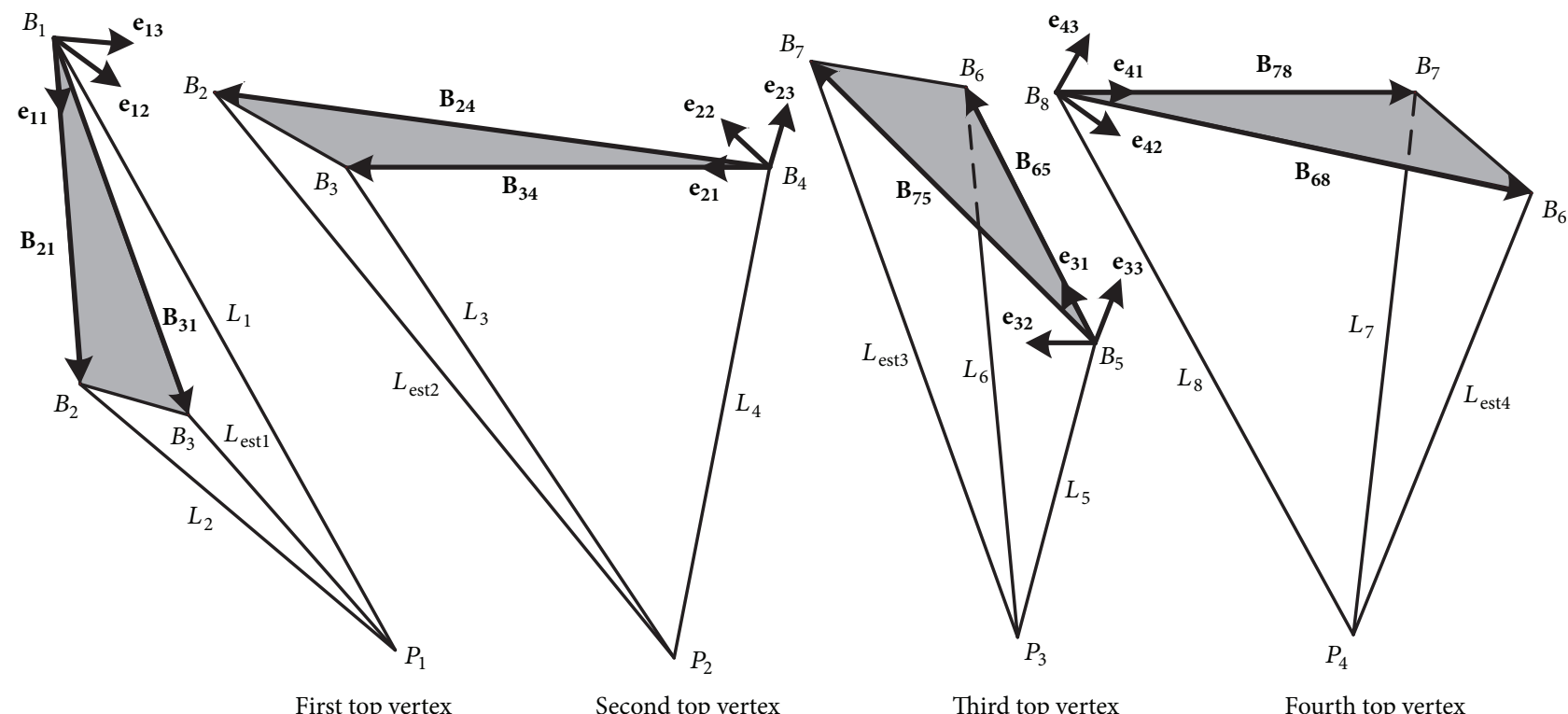

FIgURE 4: The initial value of the LM method is estimated by the approximate TA.

2.4. The Initial Selection of the LM Method. The LM method is a nonlinear least squares algorithm, the most widely used method by researchers. The key of the LM method is initial value selection. If the initial value is far away from the convergence point, the iteration speed is slow and may even result in no converging solution. According to the configuration characteristics of the CDRPR, this method estimates the initial value of the LM method based on the approximate TA.

As shown in Figure $4, \mathbf{e}_{i j}(i=1,2,3,4 ; j=1,2,3)$ are the coordinates of the tetrahedron. The first approximate tetrahedron includes two vertices $\left(P_{1}, \mathbf{B}_{21}, \mathbf{B}_{31}, B_{3}\right)$, where $P_{1}$ is the top vertex. $L_{\text {est1 }}$ is an unknown variable. Because the displacement between $B_{2}$ and $B_{3}$ is very small in the process of design, let $L_{\text {est1 }} \approx L_{2}$ in the process of solving the TA:

$$
\begin{aligned}
\lambda_{1 \_ \text {int }} & =\left\|\mathbf{P}_{1}\right\|, \\
\mathbf{P}_{1} & =\mathbf{B}_{1}+\mathbf{L}_{1}, \\
\mathbf{L}_{1} & =L_{11} \mathbf{e}_{11}+L_{12} \mathbf{e}_{12}+L_{13} \mathbf{e}_{13},
\end{aligned}
$$

where

$$
\begin{aligned}
& \mathbf{e}_{11}=\frac{\mathbf{B}_{21}}{\left\|\mathbf{B}_{21}\right\|}, \\
& \mathbf{e}_{12}=\frac{-\left(\mathbf{B}_{31} \cdot \mathbf{e}_{11}\right) \mathbf{e}_{11}+\mathbf{B}_{31}}{\left\|-\left(\mathbf{B}_{31} \cdot \mathbf{e}_{11}\right) \mathbf{e}_{11}+\mathbf{B}_{31}\right\|}, \\
& \mathbf{e}_{13}=\mathbf{e}_{11} \times \mathbf{e}_{12}, \\
& L_{11}=\frac{L_{1}^{2}+B_{21}^{2}-L_{2}^{2}}{2\left\|\mathbf{B}_{21}\right\|}, \\
& L_{12}=\frac{L_{1}^{2}+B_{31}^{2}-L_{\mathrm{est}}^{2}-2 L_{11}\left(\mathbf{B}_{31} \cdot \mathbf{e}_{11}\right)}{2\left(\mathbf{B}_{31} \cdot \mathbf{e}_{12}\right)}, \\
& L_{13}^{2}=L_{1}^{2}-L_{11}^{2}-L_{12}^{2} .
\end{aligned}
$$

$\lambda_{1 \_ \text {int }}$ is the initial value of $\lambda_{1}$.

The second approximate tetrahedron includes two vertices $\left(P_{2}, \mathbf{B}_{24}, \mathbf{B}_{34}, B_{4}\right)$, where $P_{2}$ is the top vertex. $L_{\text {est2 }}$ is an unknown variable; let $L_{\text {est } 2} \approx L_{3}$;

$$
\begin{aligned}
\lambda_{2 \_ \text {int }} & =\left\|\mathbf{P}_{2}\right\|, \\
\mathbf{P}_{2} & =\mathbf{B}_{4}+\mathbf{L}_{4}, \\
\mathbf{L}_{4} & =L_{41} \mathbf{e}_{21}+L_{42} \mathbf{e}_{22}+L_{43} \mathbf{e}_{23},
\end{aligned}
$$

where

$$
\begin{aligned}
& \mathbf{e}_{21}=\frac{\mathbf{B}_{34}}{\left\|\mathbf{B}_{34}\right\|}, \\
& \mathbf{e}_{22}=\frac{-\left(\mathbf{B}_{24} \cdot \mathbf{e}_{21}\right) \mathbf{e}_{21}+\mathbf{B}_{24}}{\left\|-\left(\mathbf{B}_{24} \cdot \mathbf{e}_{21}\right) \mathbf{e}_{21}+\mathbf{B}_{24}\right\|}, \\
& \mathbf{e}_{23}=\mathbf{e}_{21} \times \mathbf{e}_{22}, \\
& L_{41}=\frac{L_{4}^{2}+B_{34}^{2}-L_{3}^{2}}{2\left\|\mathbf{B}_{34}\right\|}, \\
& L_{42}=\frac{L_{4}^{2}+B_{24}^{2}-L_{\text {est } 2}^{2}-2 L_{41}\left(\mathbf{B}_{24} \cdot \mathbf{e}_{21}\right)}{2\left(\mathbf{B}_{24} \cdot \mathbf{e}_{22}\right)}, \\
& L_{43}^{2}=L_{4}^{2}-L_{41}^{2}-L_{42}^{2} .
\end{aligned}
$$

$\lambda_{2 \text { int }}$ is the initial value of $\lambda_{2}$.

The third approximate tetrahedron includes two vertices $\left(P_{3}, \mathbf{B}_{65}, \mathbf{B}_{75}, B_{5}\right)$, where $P_{3}$ is the top vertex. $L_{\text {est3 }}$ is an unknown variable; let $L_{\text {est } 3} \approx L_{6}$;

$$
\begin{aligned}
\lambda_{3 \_ \text {int }} & =\left\|\mathbf{P}_{3}\right\|, \\
\mathbf{P}_{3} & =\mathbf{B}_{5}+\mathbf{L}_{5}, \\
\mathbf{L}_{5} & =L_{51} \mathbf{e}_{31}+L_{52} \mathbf{e}_{32}+L_{53} \mathbf{e}_{33},
\end{aligned}
$$


where

$$
\begin{aligned}
& \mathbf{e}_{31}=\frac{\mathbf{B}_{65}}{\left\|\mathbf{B}_{65}\right\|}, \\
& \mathbf{e}_{32}=\frac{-\left(\mathbf{B}_{75} \cdot \mathbf{e}_{31}\right) \mathbf{e}_{31}+\mathbf{B}_{75}}{\left\|-\left(\mathbf{B}_{75} \cdot \mathbf{e}_{31}\right) \mathbf{e}_{31}+\mathbf{B}_{75}\right\|}, \\
& \mathbf{e}_{33}=\mathbf{e}_{31} \times \mathbf{e}_{32}, \\
& L_{51}=\frac{L_{5}^{2}+B_{65}^{2}-L_{6}^{2}}{2\left\|\mathbf{B}_{65}\right\|}, \\
& L_{52}=\frac{L_{5}^{2}+B_{75}^{2}-L_{\text {est3 }}^{2}-2 L_{51}\left(\mathbf{B}_{75} \cdot \mathbf{e}_{31}\right)}{2\left(\mathbf{B}_{75} \cdot \mathbf{e}_{32}\right)}, \\
& L_{53}^{2}=L_{5}^{2}-L_{51}^{2}-L_{52}^{2} .
\end{aligned}
$$

$\lambda_{3 \text { int }}$ is the initial value of $\lambda_{3}$.

The fourth approximate tetrahedron includes two vertices $\left(P_{4}, \mathbf{B}_{68}, \mathbf{B}_{78}, B_{8}\right)$, where $P_{4}$ is the top vertex. $L_{\text {est } 4}$ is an unknown variable; let $L_{\text {est } 4} \approx L_{7}$;

$$
\begin{aligned}
\lambda_{4 \_ \text {int }} & =\left\|\mathbf{P}_{4}\right\|, \\
\mathbf{P}_{4} & =\mathbf{B}_{8}+\mathbf{L}_{8}, \\
\mathbf{L}_{8} & =L_{81} \mathbf{e}_{41}+L_{82} \mathbf{e}_{42}+L_{83} \mathbf{e}_{43},
\end{aligned}
$$

where

$$
\begin{aligned}
& \mathbf{e}_{41}=\frac{\mathbf{B}_{78}}{\left\|\mathbf{B}_{78}\right\|}, \\
& \mathbf{e}_{42}=\frac{-\left(\mathbf{B}_{68} \cdot \mathbf{e}_{41}\right) \mathbf{e}_{41}+\mathbf{B}_{68}}{\left\|-\left(\mathbf{B}_{68} \cdot \mathbf{e}_{41}\right) \mathbf{e}_{41}+\mathbf{B}_{68}\right\|}, \\
& \mathbf{e}_{43}=\mathbf{e}_{41} \times \mathbf{e}_{42}, \\
& L_{81}=\frac{L_{8}^{2}+B_{78}^{2}-L_{7}^{2}}{2\left\|\mathbf{B}_{78}\right\|}, \\
& L_{82}=\frac{L_{8}^{2}+B_{68}^{2}-L_{\text {est }}^{2}-2 L_{81}\left(\mathbf{B}_{68} \cdot \mathbf{e}_{41}\right)}{2\left(\mathbf{B}_{68} \cdot \mathbf{e}_{42}\right)}, \\
& L_{83}^{2}=L_{8}^{2}-L_{81}^{2}-L_{82}^{2} .
\end{aligned}
$$

$\lambda_{4 \text { int }}$ is the initial value of $\lambda_{4}$.

$\mathbf{B}_{i j}=\mathbf{B}_{i}-\mathbf{B}_{j}$; it is a known parameter. If the length of the cable is measured by the cable-length sensor, the iterative initial value of (9) is obtained from (14)-(22). Since the initial value of $\lambda_{i}$ is close to the convergence value, the number of iterations is finite, and the FK problem can be solved in realtime.

2.5. Numerical Example for the FK. To illustrate the convergence of the LM method for FK problem, a numerical example is given. The overhead view of the CDRPR is shown in Figure 5 and the geometric parameters of the CDRPR are shown in Table 1 . The termination threshold of the LM algorithm is chosen to be $10^{-6}$. The maximum number of
TABLE 1: Geometric parameters of the CDRPR.

\begin{tabular}{lccc}
\hline Parameter & Value $(\mathrm{m})$ & Parameter & Value $(\mathrm{m})$ \\
\hline$l_{B 1}$ & 1.776 & $w_{B 2}$ & 0.105 \\
$l_{B 2}$ & 1.776 & $w_{P}$ & 0.180 \\
$w_{B 1}$ & 0.105 & $l_{P}$ & 0.480 \\
\hline
\end{tabular}

TABLE 2: The vector parameters of the CDRPR.

\begin{tabular}{lc}
\hline Vector & Value $(\mathrm{m})$ \\
\hline $\mathbf{B}_{\mathbf{1}}$ & {$[0.888,0.053,0]^{T}$} \\
$\mathbf{B}_{2}$ & {$[0.053,0.888,0]^{T}$} \\
$\mathbf{B}_{3}$ & {$[-0.053,0.888,0]^{T}$} \\
$\mathbf{B}_{4}$ & {$[-0.888,0.053,0]^{T}$} \\
$\mathbf{B}_{5}$ & {$[-0.888,-0.053,0]^{T}$} \\
$\mathbf{B}_{6}$ & {$[-0.053,-0.888,0]^{T}$} \\
$\mathbf{B}_{7}$ & {$[0.053,-0.888,0]^{T}$} \\
$\mathbf{B}_{8}$ & {$[0.888,-0.053,0]^{T}$} \\
$\mathbf{p}_{\mathbf{1}}$ & {$[0.090,0.240,0.098]^{T}$} \\
$\mathbf{p}_{2}$ & {$[-0.090,0.240,0.098]^{T}$} \\
$\mathbf{p}_{3}$ & {$[-0.090,-0.240,0.098]^{T}$} \\
$\mathbf{p}_{4}$ & {$[0.090,-0.240,0.098]^{T}$} \\
\hline
\end{tabular}

iterations is set to 50. The vector parameters of the CDRPR are shown in Table 2.

First, the data of cable length is produced by (2) based on the inverse of the CDRPR, shown in Table 3. Then, the approximate tetrahedron approach is used to obtain an initial iterate for LM method via (14)-(22). Equation (9) derived by TA is solved using the LM method based on the initial iterate. The initial value of $\lambda_{1}, \lambda_{2}, \lambda_{3}, \lambda_{4}$ and convergence from this initial iterate toward the true solution are shown in Figure 6. The FK of the CDRPR could be obtained via (10), (11), and (13) based on the solutions of (9).

As shown in Figure 6, (a), (b), (c), and (d) of Figure 6 show the convergence of $\lambda_{1}, \lambda_{2}, \lambda_{3}, \lambda_{4}$ in the six directions, respectively. The error between the actual value and ideal value of $\lambda_{i}$ converges to zero in finite step. The starting point on the figures is the distance from the initial value to the true solution. The figures show that $\lambda_{i}$ could converge to the true solutions in finite step based on the suitable initial value of the LM method. It means that the algorithm of initial selection for the LM method is effective. The FK of the CDRPR could be solved immediately if the data of the cable length were obtained by the cable-length sensor. The control system could control the pose of the end-effector in real-time based on the feedback of the FK.

\section{Dynamic}

The cable mass is very small compared with the end-effector, and the rigidity is larger. Therefore, the quality and elasticity of the rope is ignored. The dynamic model of the robot is simplified as the end-effector model. The platform is fixed in the inertial coordinate system since the movement of the supply ship is very small at sea. 

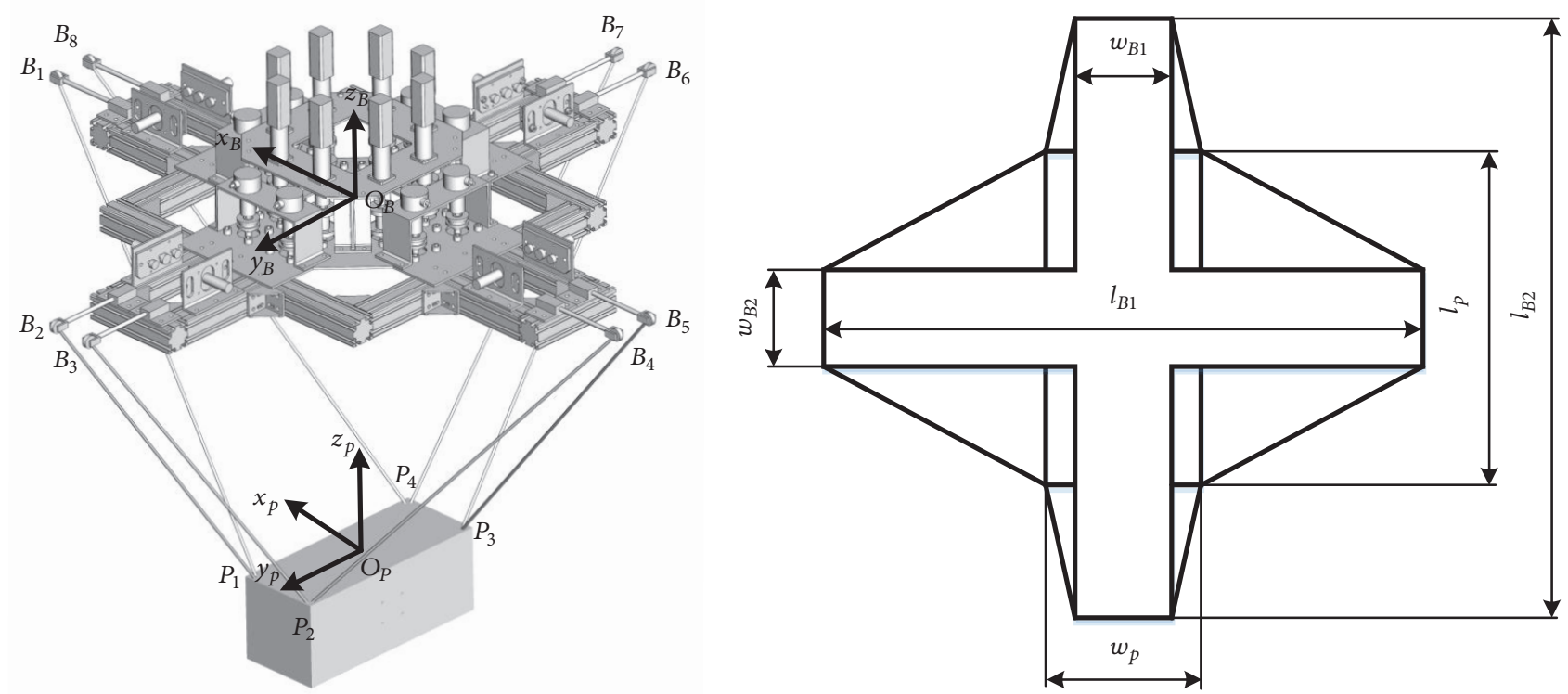

FIgure 5: The overhead view of the CDRPR.
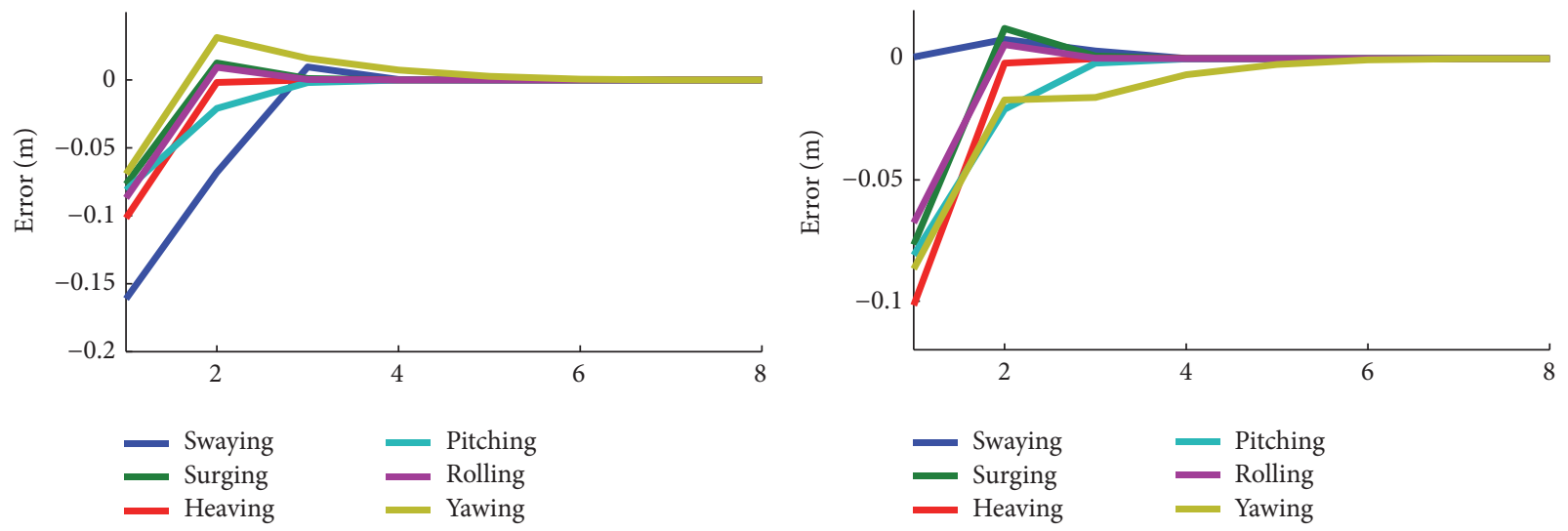

(a) Number of iterations
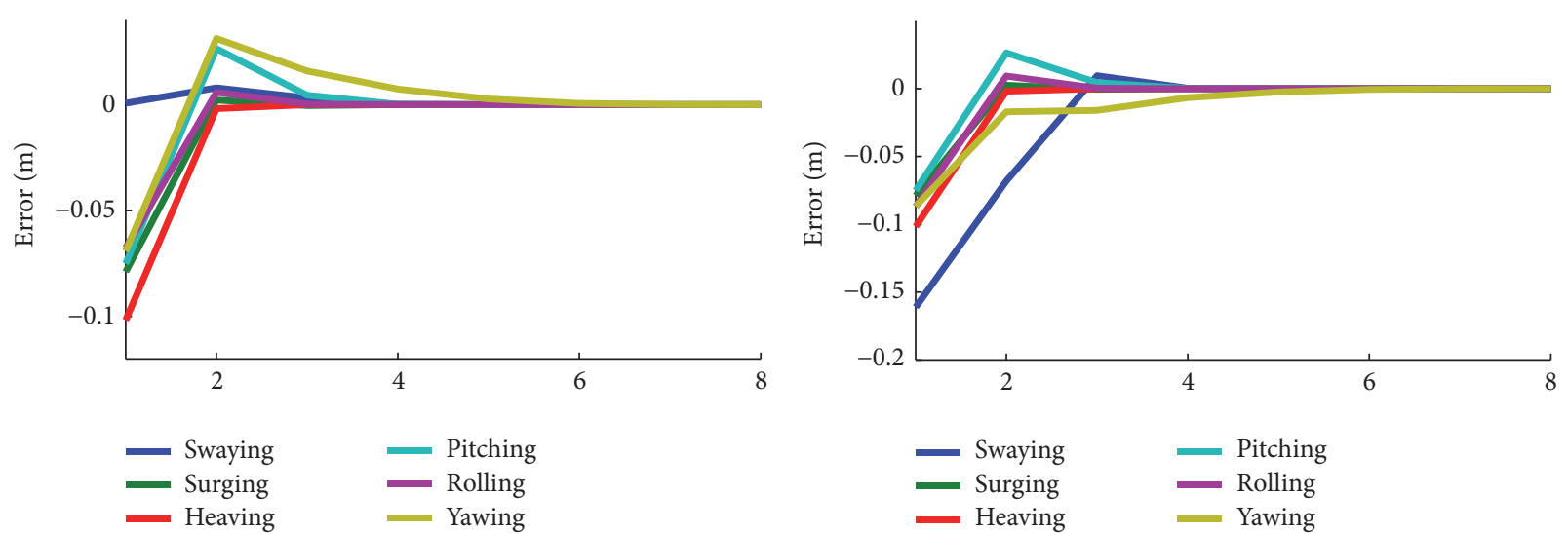

$$
\begin{array}{ll}
\text { Swaying } & \text { Pitching } \\
\text { - Surging } & \text { Rolling }
\end{array}
$$

(c) Number of iterations

(d) Number of iterations

FIgURE 6: The convergence from initial iterate toward the true solution. 
TABLE 3: The data of cable length in six directions.

\begin{tabular}{lcccccccc}
\hline Direction & $L_{1}(\mathrm{~m})$ & $L_{2}(\mathrm{~m})$ & $L_{3}(\mathrm{~m})$ & $L_{4}(\mathrm{~m})$ & $L_{5}(\mathrm{~m})$ & $L_{6}(\mathrm{~m})$ & $L_{7}(\mathrm{~m})$ & $L_{8}(\mathrm{~m})$ \\
\hline Swaying & 1.317 & 1.279 & 1.274 & 1.423 & 1.423 & 1.274 & 1.279 & 1.317 \\
Surging & 1.379 & 1.240 & 1.240 & 1.379 & 1.360 & 1.309 & 1.309 & 1.360 \\
Heaving & 1.177 & 1.066 & 1.066 & 1.177 & 1.177 & 1.066 & 1.066 \\
Pitching & 1.336 & 1.251 & 1.251 & 1.336 & 1.401 & 1.301 & 1.301 \\
Rolling & 1.371 & 1.283 & 1.264 & 1.367 & 1.367 & 1.264 & 1.283 \\
Yawing & 1.374 & 1.271 & 1.275 & 1.362 & 1.374 & 1.271 & 1.401 \\
\hline
\end{tabular}

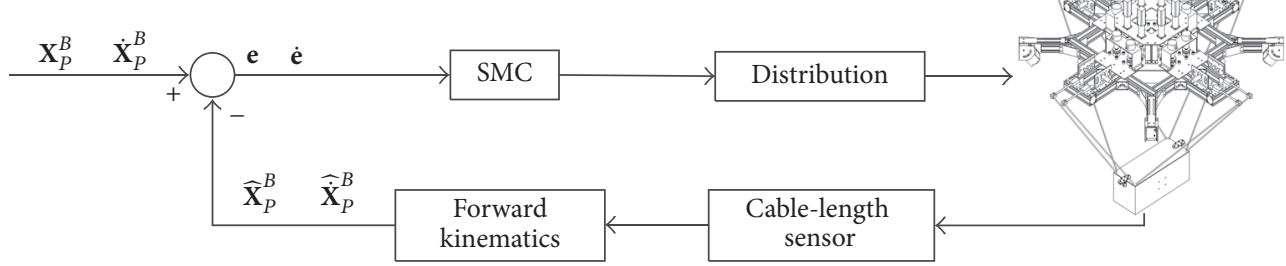

Figure 7: Block diagram of the robot control system.

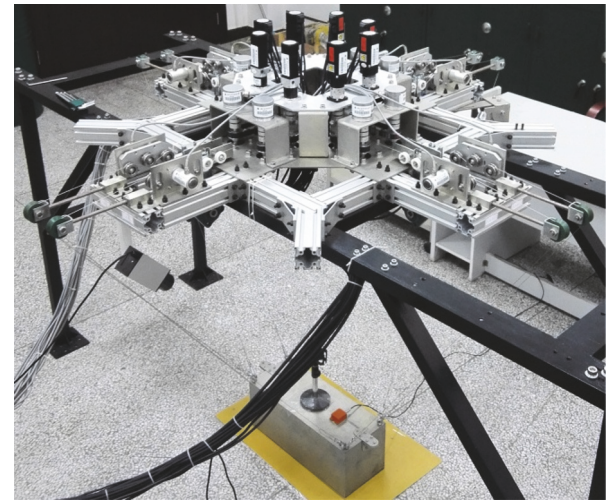

FIGURE 8: Principle prototype of CDRPR.

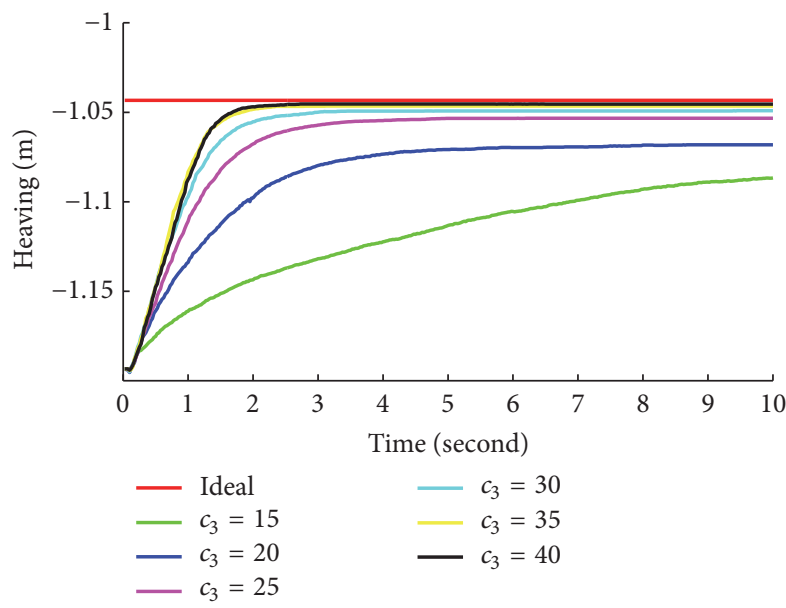

FIGURE 9: Tracking performance of the system in different sliding surface parameters.

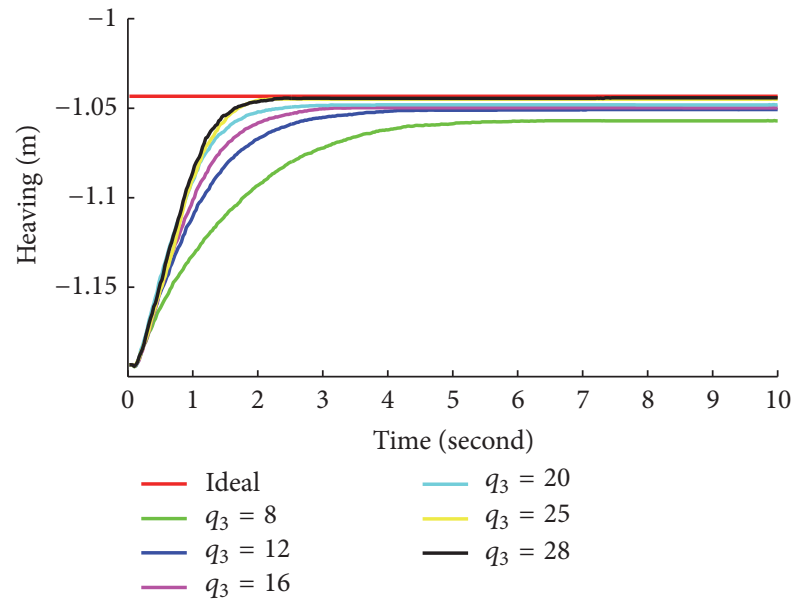

FIGURE 10: Tracking performance of the system in different reaching speed parameters.

According to the Newton equation

$$
m \ddot{\mathbf{x}}_{P}^{B}=\mathbf{f}+m \mathbf{g}+\mathbf{f}_{\mathrm{ext}},
$$

where $m$ is the end-effector mass and $\mathbf{g}$ is the gravity vector. $\mathbf{f}$ represents the vector of the resultant force of rope on the endeffector. $\mathbf{f}_{\text {ext }}$ is the vector of the resultant force of the external environment on the end-effector.

According to the Euler equation

$$
\mathbf{I} \dot{\boldsymbol{\omega}}_{P}^{B}+\boldsymbol{\omega}_{P}^{B} \times\left(\mathbf{I} \boldsymbol{\omega}_{P}^{B}\right)=\mathbf{m}+\mathbf{m}_{\mathrm{ext}},
$$

where $\mathbf{I}$ is an end-effector inertia matrix relative to the centroid. $\mathbf{m}$ represents the vector of the resultant moment of 

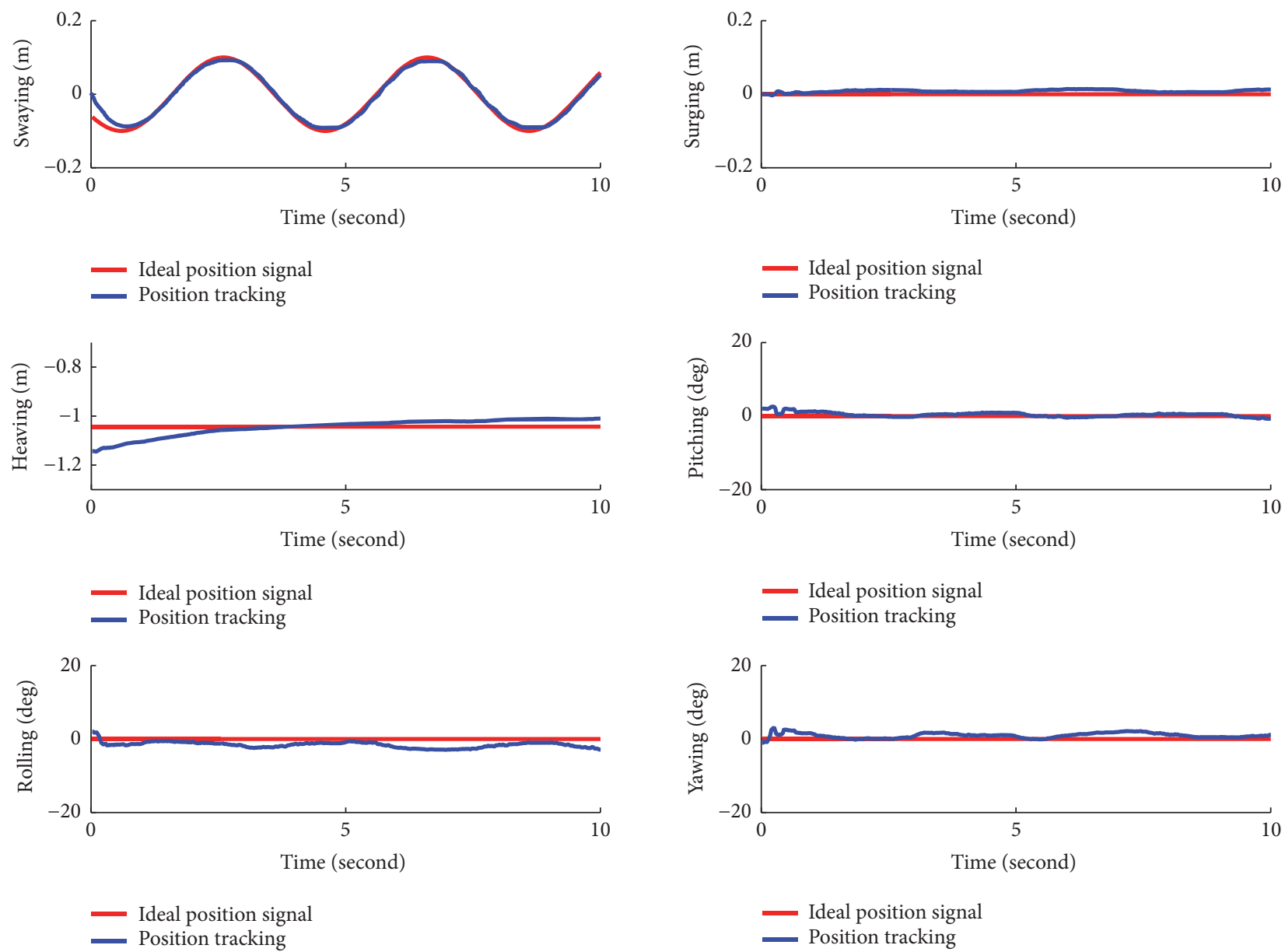

(a)
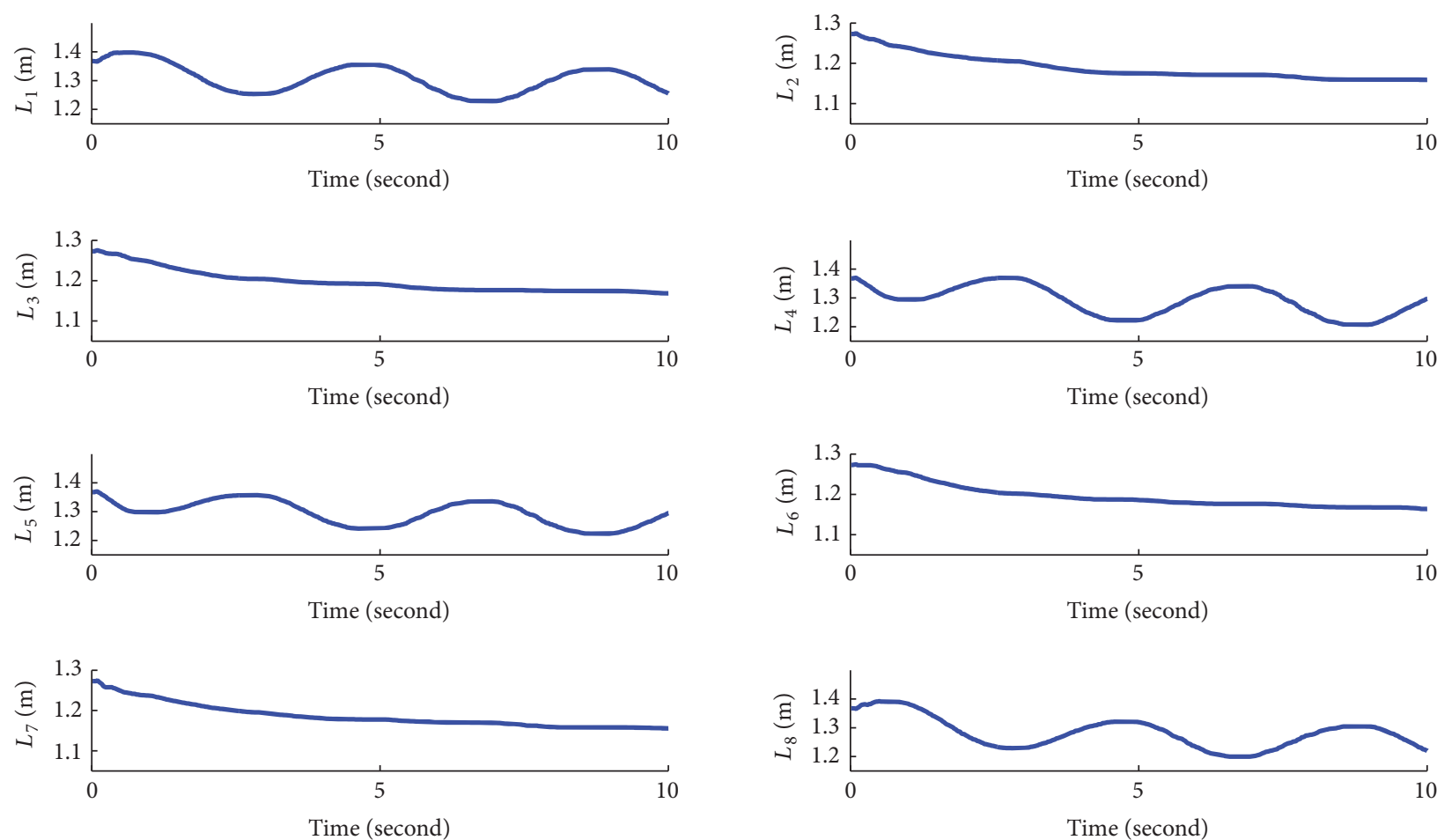

(b)

Figure 11: Continued. 

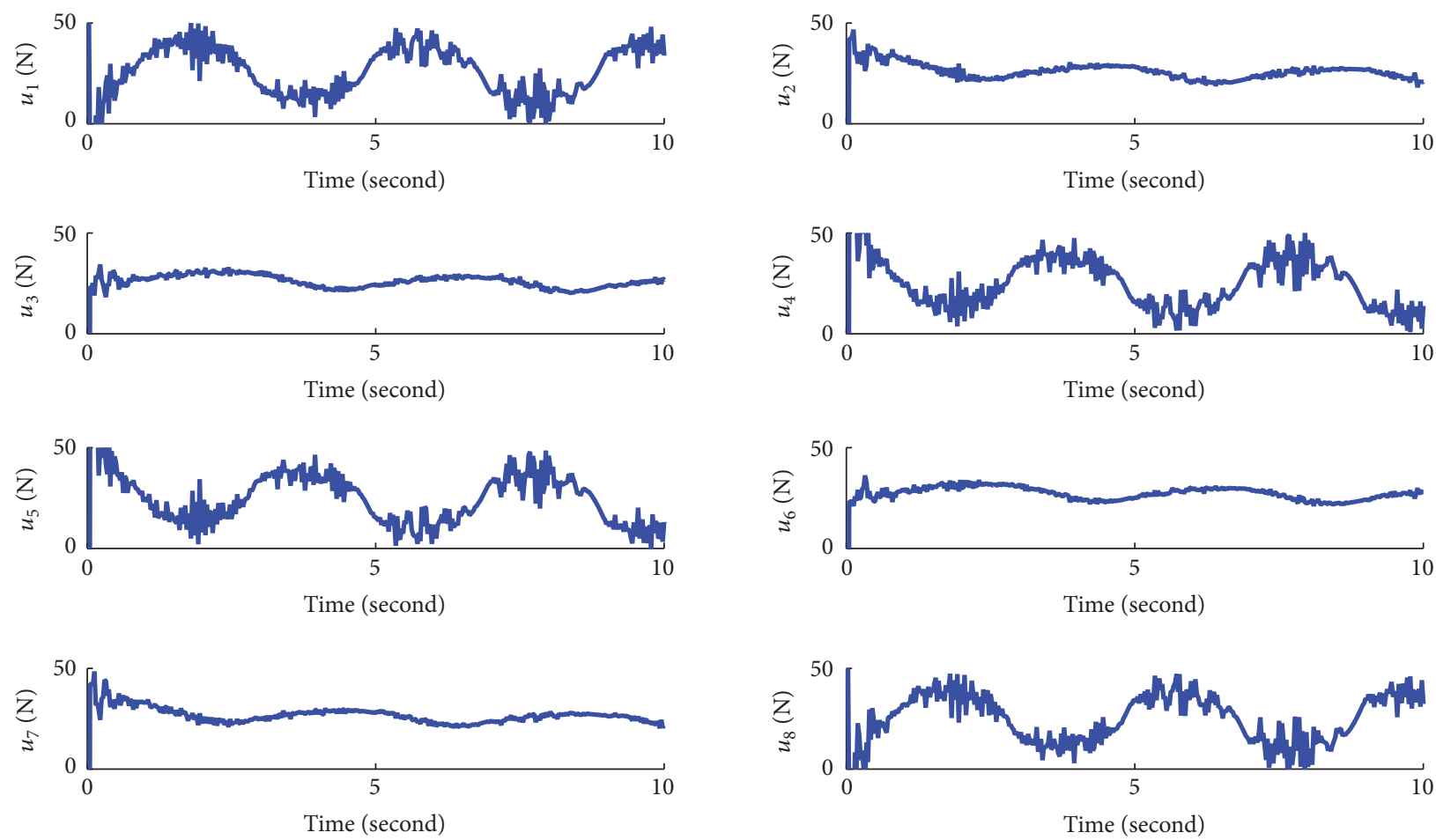

(c)

FIGURE 11: Tracking in swaying direction.

rope on the end-effector. $\mathbf{m}_{\text {ext }}$ is the vector of the resultant moment of external environment on the end-effector.

Combining (23) with (24), the dynamic of end-effector is

$$
\mathbf{M} \ddot{\mathbf{X}}_{P}^{B}+\mathbf{C} \dot{\mathbf{X}}_{P}^{B}-\mathbf{G}-\mathbf{D}=\mathbf{J}^{T} \mathbf{u}
$$

where $\mathbf{M}=\left[\begin{array}{cc}m \mathbf{E}_{3 \times 3} & \mathbf{0}_{3 \times 3} \\ \mathbf{0}_{3 \times 3} & \mathbf{I}\end{array}\right], \mathbf{C}=\left[\begin{array}{cc}\mathbf{0}_{3 \times 3} & \mathbf{0}_{3 \times 3} \\ \mathbf{0}_{3 \times 3} & {\left[\boldsymbol{\omega}_{P B}^{B} \times\right] \mathbf{I}}\end{array}\right], \mathbf{G}=\left[\begin{array}{c}m \mathbf{g} \\ \mathbf{0}_{3 \times 1}\end{array}\right]$, and $\mathbf{D}=\left[\begin{array}{c}\mathbf{f}_{\text {ext }} \\ \mathbf{m}_{\text {ext }}\end{array}\right] . \mathbf{J}^{T}$ is the Jacobian matrix of the robot.

\section{Control}

In this paper, the sliding mode method is designed based on the Lyapunov function. The sliding mode control has the advantages of fast response, insensitivity to parameter variation and disturbance, and simple physical realization. Figure 7 shows the block diagram for the robot control system. The target position and velocity of the end-effector relative to the end-effector is set up. The FK proposed in this paper is used to compute the actual position and velocity in real-time based on the length of the cable measured by the cable-length sensor. The error between the detection value and the target value is regarded as an input of the control system. The rope tension is computed by the sliding mode control and tension distribution method. The servo systems control the end-effector motion through the rope tension.
The sliding surface and Lyapunov functions are

$$
\begin{aligned}
\mathbf{S} & =\left[\begin{array}{lll}
s_{1} & \cdots & s_{6}
\end{array}\right]^{T}=\dot{\mathbf{X}}_{P}^{B}-\widehat{\mathbf{X}}_{P}^{B}+\mathbf{C}_{e}\left(\mathbf{X}_{P}^{B}-\widehat{\mathbf{X}}_{P}^{B}\right), \\
V & =\frac{1}{2} \mathbf{S}^{T} \mathbf{S},
\end{aligned}
$$

where $\mathbf{X}_{P}^{B}$ and $\dot{\mathbf{X}}_{P}^{B}$ are the ideal position and velocity of the end-effector relative to the base platform, respectively. $\widehat{\mathbf{X}}_{P}^{B}$ and $\widehat{\dot{\mathbf{x}}}^{B}$

$\dot{\mathbf{X}}_{P}$ are the actual position and velocity of the end-effector relative to the base platform measured by the cable-length sensor, respectively. $\mathbf{C}_{e}=\operatorname{diag}\left(c_{1}, \ldots, c_{6}\right)$.

$$
\begin{aligned}
\dot{V} & =\mathbf{S}^{T} \dot{\mathbf{S}}=\mathbf{S}^{T}\left(-\mathbf{M}^{-1}\left(\mathbf{J}^{T} \mathbf{u}-\mathbf{C X}_{P}^{B}+\mathbf{G}+\mathbf{D}\right)+\ddot{\mathbf{X}}_{P}^{B}\right. \\
& \left.+\mathbf{C}_{e}\left(\dot{\mathbf{X}}_{P}^{B}-\widehat{\dot{\mathbf{X}}}_{P}^{B}\right)\right) .
\end{aligned}
$$

Exponential approach law is used:

$$
\dot{s}=-k \operatorname{sgn}(\mathbf{s})-q \mathbf{s} \quad k>0, q>0,
$$

where $\operatorname{sgn}(\cdot)$ represents the symbolic function

$$
\operatorname{sgn}(s)= \begin{cases}1, & s>0 \\ 0, & s=0 \\ -1, & s<0 .\end{cases}
$$



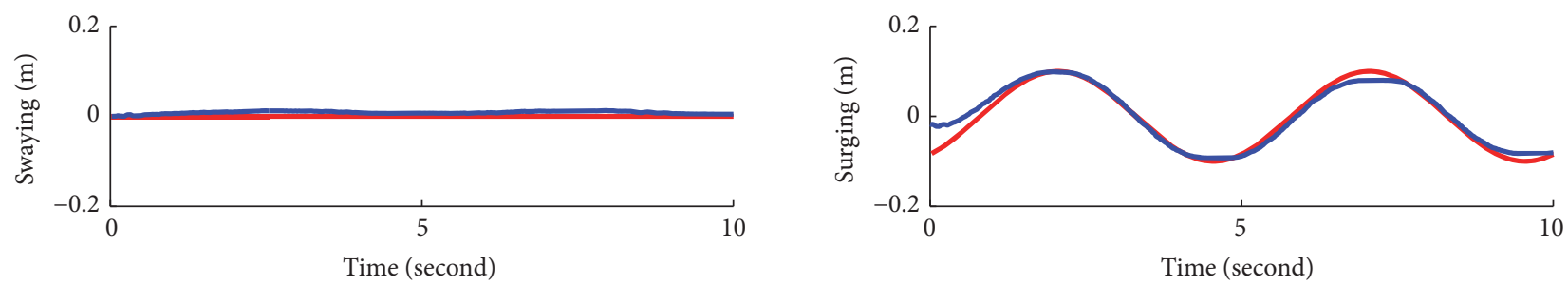

Ideal position signal

Position tracking
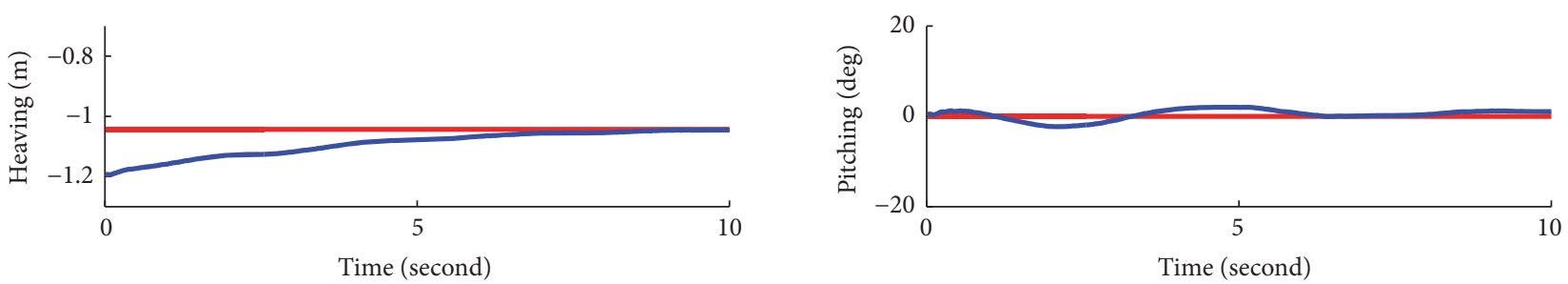

Ideal position signal

Position tracking
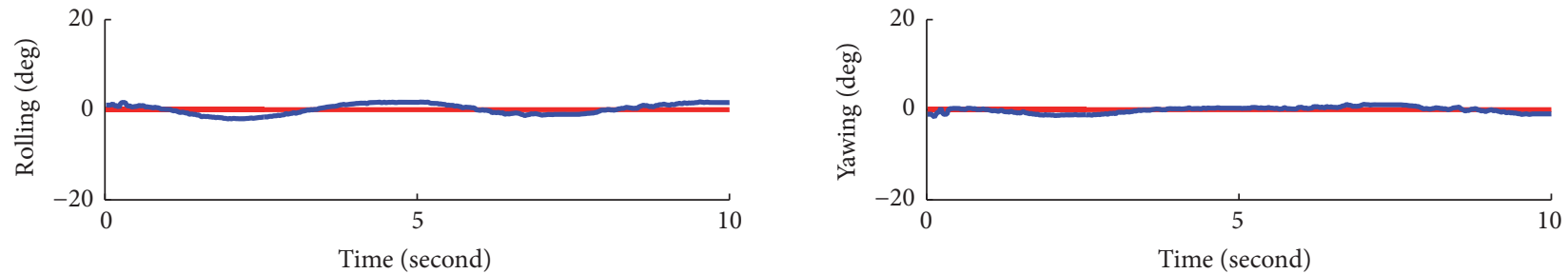

Ideal position signal

Position tracking

(a)
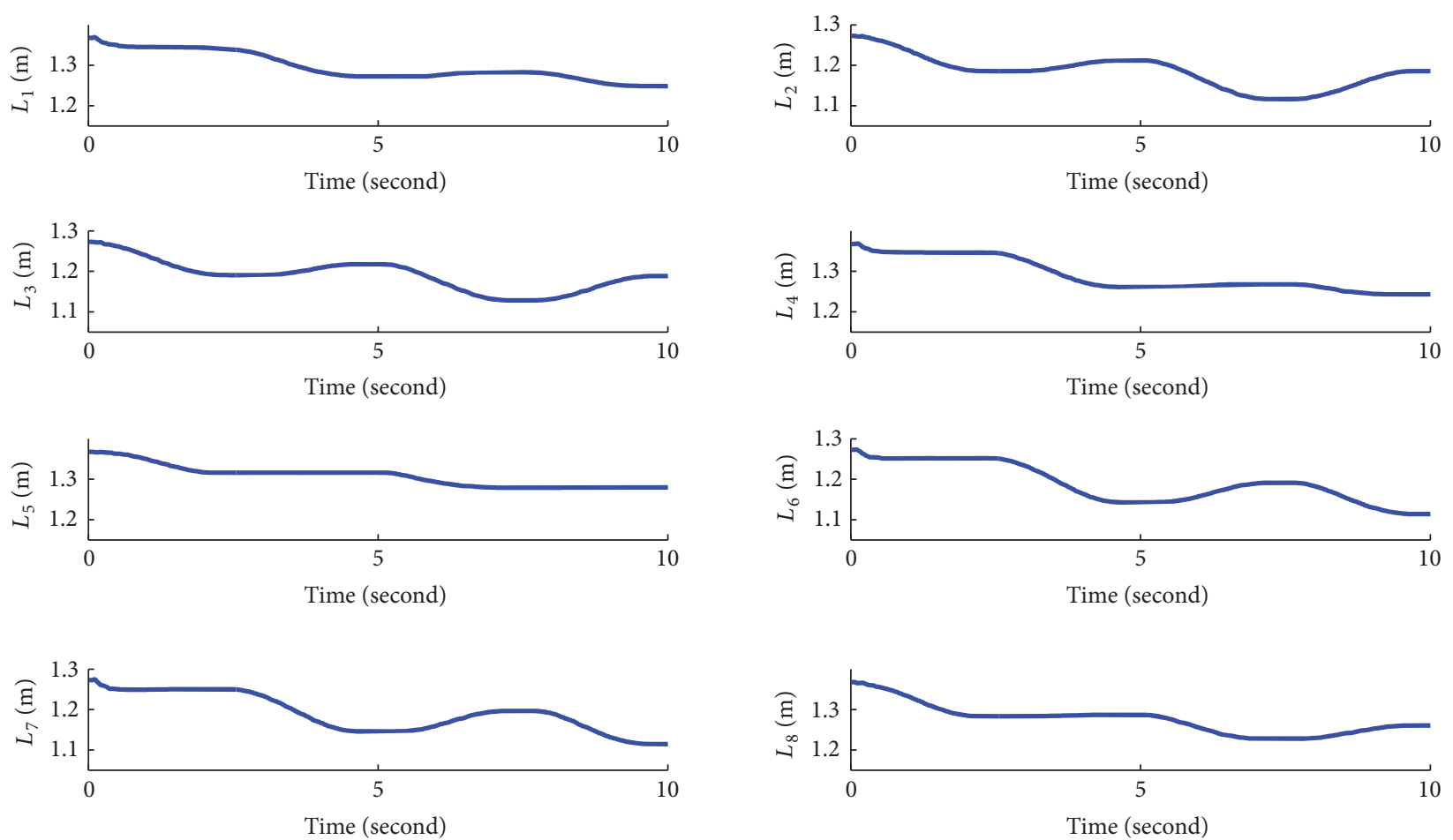

(b)

FIGURE 12: Continued. 

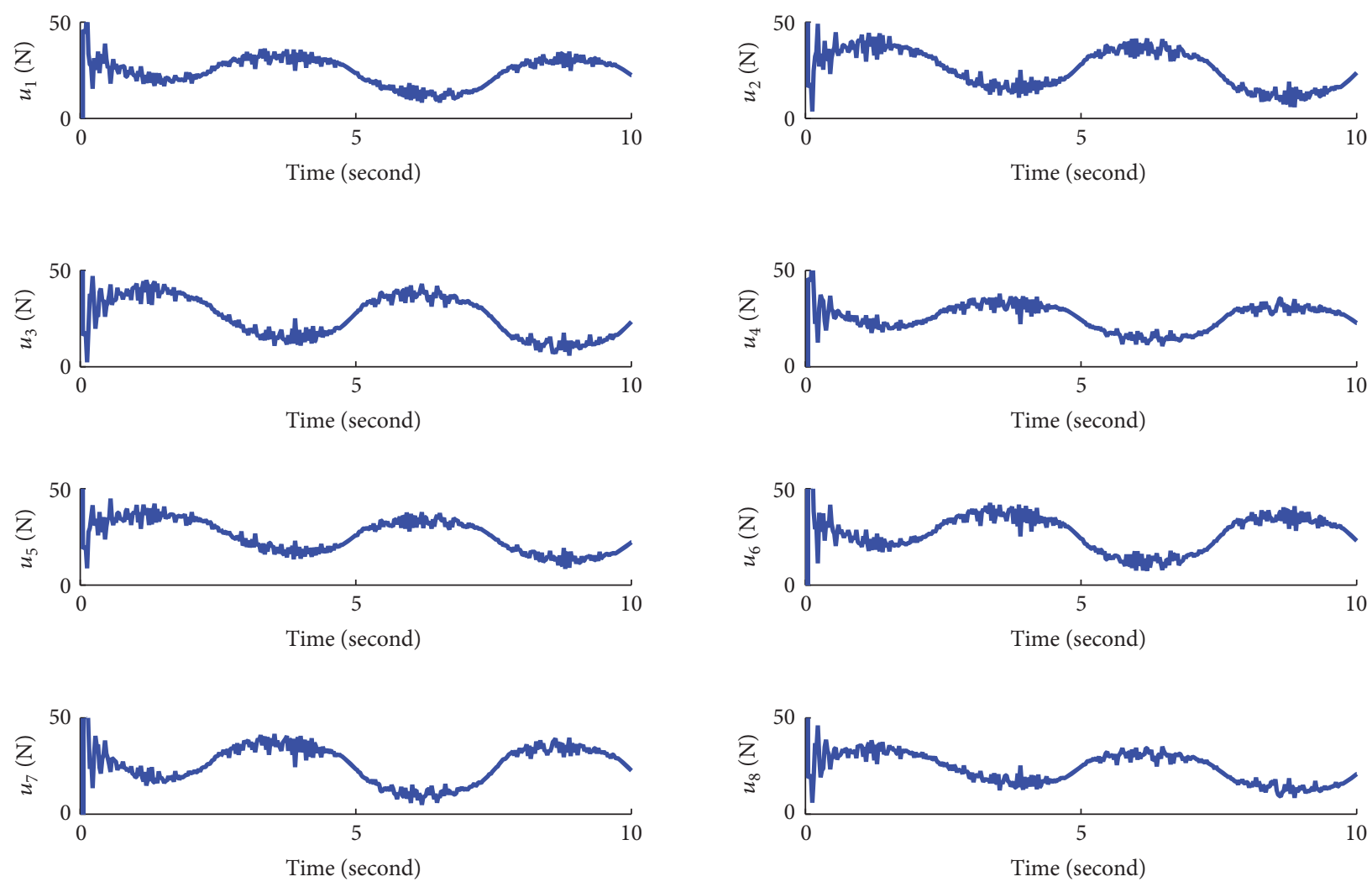

(c)

FIgURE 12: Tracking in a surging direction.

In order to ensure that $\dot{V}$ is negative and the disturbance is unknown, the interference bound is used to design the control law

$$
\begin{aligned}
\mathbf{J}^{T} \mathbf{u}= & \mathbf{M}\left(\ddot{\mathbf{X}}_{P}^{B}+\mathbf{C}_{\boldsymbol{e}}\left(\dot{\mathbf{X}}_{P}^{B}-\hat{\mathbf{X}}_{P}^{B}\right)+\mathbf{K} \operatorname{sgn}(\mathbf{s})+\mathbf{Q s}\right) \\
& +\mathbf{C} \dot{\mathbf{X}}_{P}^{B}-\mathbf{G}-\mathbf{D}_{c},
\end{aligned}
$$

where $\mathbf{K}=\operatorname{diag}\left(k_{1}, \ldots, k_{6}\right)$ and $\mathbf{Q}=\operatorname{diag}\left(q_{1}, \ldots, q_{6}\right)$. According to (30), $\dot{V}$ is

$$
\dot{V}=\mathbf{S}^{T}\left(-\mathbf{K} \operatorname{sgn}(\mathbf{s})-\mathbf{Q} \mathbf{s}-\mathbf{M}^{-1}\left(\mathbf{D}_{c}-\mathbf{D}\right)\right) .
$$

One has hypothesis

$$
\mathbf{D}_{L} \leq \mathbf{D} \leq \mathbf{D}_{U}
$$

where $\mathbf{D}_{L}$ and $\mathbf{D}_{U}$ are the disturbance bound; let

$$
\mathbf{D}_{c}=\mathbf{D}_{2}-\mathbf{D}_{1} \operatorname{sgn}(\mathbf{s}),
$$

where $\mathbf{D}_{1}=\left(\mathbf{D}_{U}-\mathbf{D}_{L}\right) / 2$ and $\mathbf{D}_{2}=\left(\mathbf{D}_{U}+\mathbf{D}_{L}\right) / 2$, which is to ensure $\dot{V} \leq 0$. The system is globally asymptotically stable.
To avoid system flutter, the symbol function $\operatorname{sgn}(\cdot)$ is replaced with saturation function sat $(\cdot)$ :

$$
\text { sat }(s)= \begin{cases}1 & s>\Delta \\ \frac{s}{\Delta} & |s| \leq \Delta \\ -1 & s<-\Delta .\end{cases}
$$

At this point, the sliding mode control law is

$$
\begin{aligned}
\mathbf{J}^{T} \mathbf{u}= & \mathbf{M}\left(\ddot{\mathbf{X}}_{P}^{B}+\mathbf{C}_{e}\left(\dot{\mathbf{X}}_{P}^{B}-\hat{\mathbf{X}}_{P}^{B}\right)+\mathbf{K} \operatorname{sat}(\mathbf{s})+\mathbf{Q s}\right) \\
& +\mathbf{C} \dot{\mathbf{X}}_{P}^{B}-\mathbf{G}-\mathbf{D}_{c} .
\end{aligned}
$$

A reasonable control law can be designed as long as the range of $\mathbf{D}$ is known, which makes the system stable and asymptotically convergent to $\mathbf{S}=\dot{\mathbf{S}}=\mathbf{0}$; the robot is based on redundant parallel mechanism. The method of tension distribution optimization should be used to improve control accuracy. The interactive projection tension distribution optimization method [37] was used in this paper.

\section{Experimental}

To verify the effectiveness of the proposed cable-length sensor feedback method for the control scheme, simulations and 

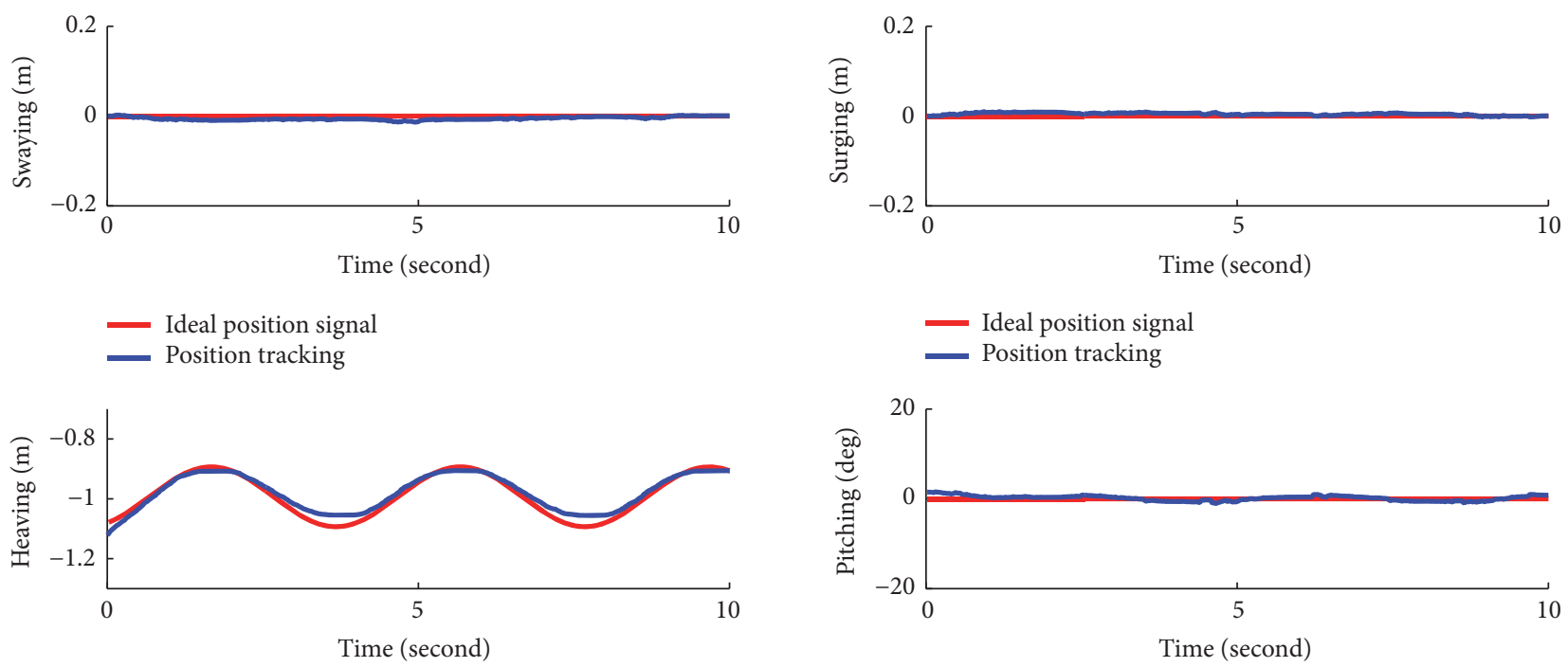

deal position signal Position tracking
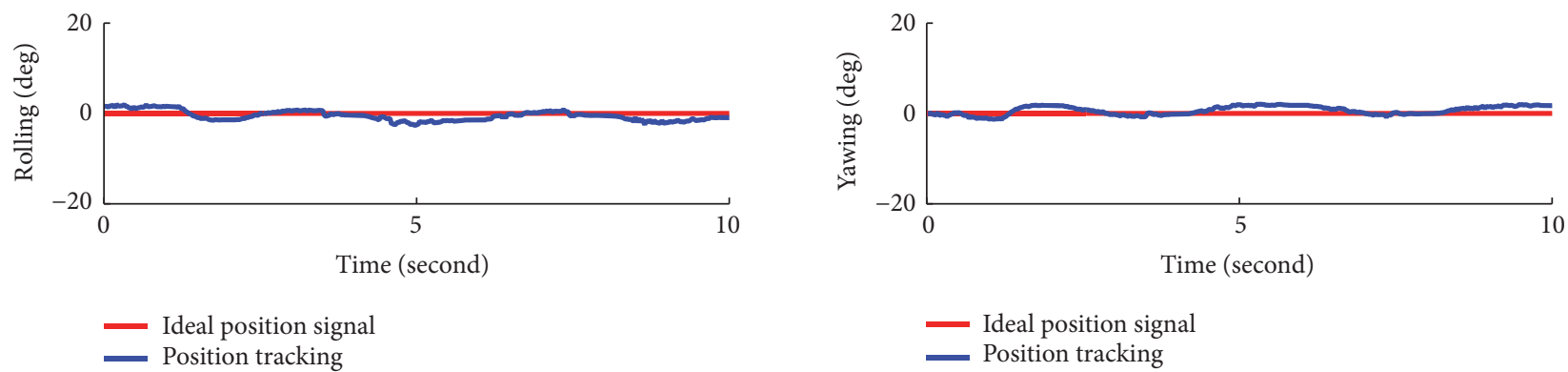

(a)
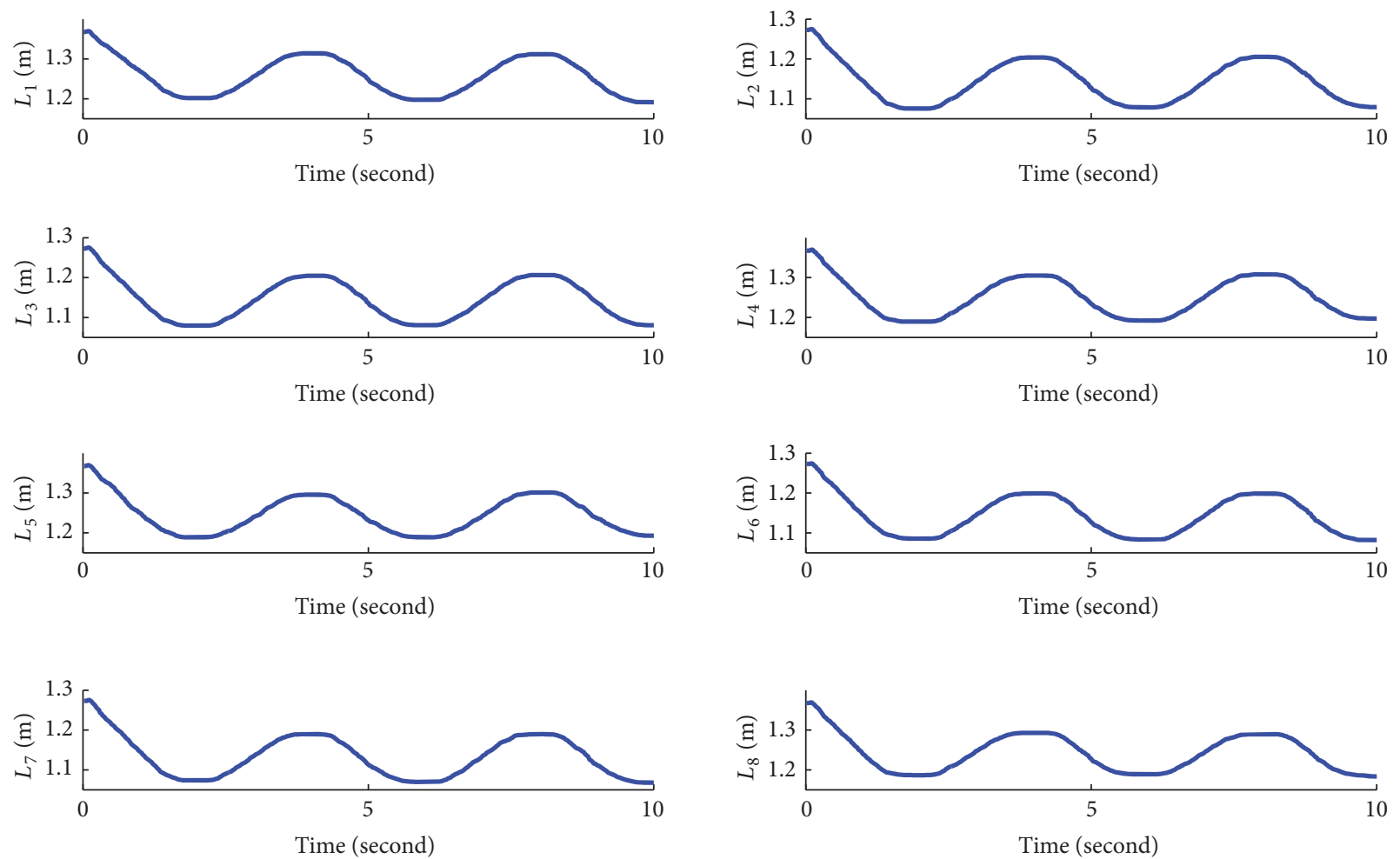

(b)

Figure 13: Continued. 

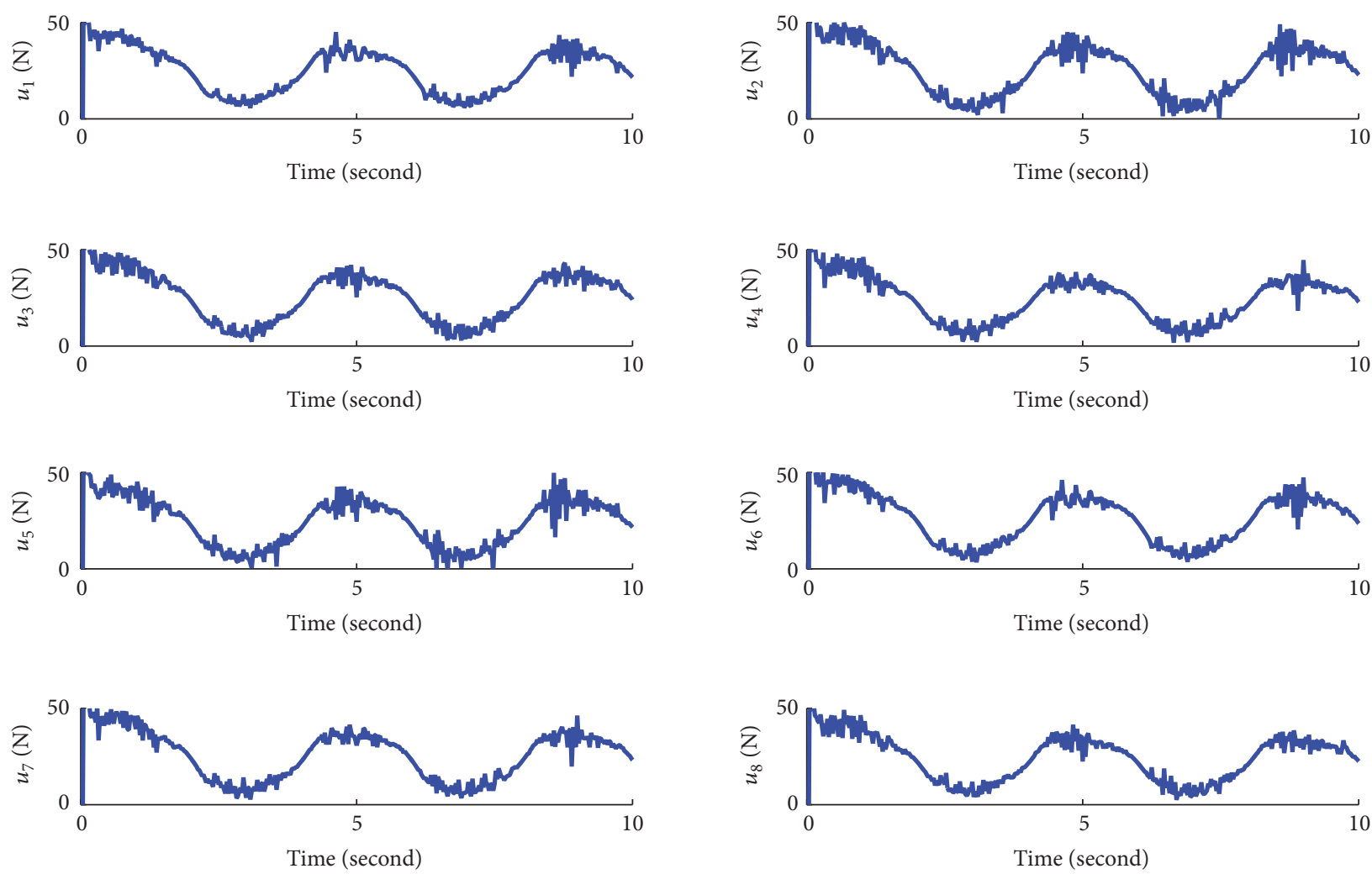

(c)

FIGURE 13: Tracking in a heaving direction.

experiments are applied to CDRPR. The principle prototype of CDRPR is shown in Figure 8. The controller solves the FK according to the length measured by the cable-length sensor using the method proposed in this article. The geometric notation is shown in Figure 5(b). The structural parameter of CDRPR is shown in Table 1. The end-effector mass is $m=$ $16.576 \mathrm{~kg}$. The control system is established based on the $\mathrm{C}++$ language.

The first experiment is to study the influence of the control parameters on the control performance. Sliding surface parameter $\mathbf{C}_{e}$ and reaching speed parameter $\mathbf{Q}$ have great influence on control performance. Given a step signal, the tracking performance of different parameters is observed in a heaving direction.

First, the step signal is $\mathbf{X}_{P}^{B}=\left[\begin{array}{llllll}0 & 0 & -1.04 & 0 & 0 & 0\end{array}\right]^{T}$, the control parameter $K=\operatorname{diag}\left(\begin{array}{llllll}2 & 2 & 2 & 2 & 2 & 2\end{array}\right)^{T}$, $\Delta=5, C_{e}=\operatorname{diag}\left(\begin{array}{llllll}15 & 15 & c_{3} & 15 & 15 & 15\end{array}\right)^{T}$, and $Q=\operatorname{diag}\left(\begin{array}{llllll}8 & 8 & 8 & 8 & 8 & 8\end{array}\right)^{T}$. The initial value of $\widehat{\mathbf{X}}_{P}^{B}$ is $\widehat{\mathbf{X}}_{P}^{B}=\left[\begin{array}{llllll}0 & 0 & -1.19 & 0 & 0 & 0\end{array}\right]^{T}$. Other parameters are fixed. Let $c_{3}=15, c_{3}=20, c_{3}=25, c_{3}=30, c_{3}=35$, and $c_{3}=40$. The tracking results are obtained in different parameters in the step signal, as shown in Figure 9.

Second, the step signal is $\mathbf{X}_{P}^{B}=\left[\begin{array}{llllll}0 & 0 & -1.04 & 0 & 0 & 0\end{array}\right]^{T}$, the control parameter $K=\operatorname{diag}\left(\begin{array}{llllll}2 & 2 & 2 & 2 & 2 & 2\end{array}\right)^{T}$,
$\Delta=5, C_{e}=\operatorname{diag}\left(\begin{array}{llllll}15 & 15 & 25 & 15 & 15 & 15\end{array}\right)^{T}$, and $Q=$ $\operatorname{diag}\left(\begin{array}{llllll}8 & 8 & q_{3} & 8 & 8 & 8\end{array}\right)^{T}$. The initial value of $\widehat{\mathbf{X}}_{P}^{B}$ is $\widehat{\mathbf{X}}_{P}^{B}=$ $\left[\begin{array}{llllll}0 & 0 & -1.19 & 0 & 0 & 0\end{array}\right]^{T}$. Other parameters are fixed. Let $q_{3}=8, q_{3}=12, q_{3}=16, q_{3}=20, q_{3}=25$, and $q_{3}=28$. The tracking results are obtained in different parameters in the step signal, as shown in Figure 10.

It can be seen that, with the increase of $c_{3}$, the faster the response of sliding mode, the more rapid the performance. With the increase of $q_{3}$, the system reaches the sliding surface more rapidly. However, the tracking performance is not greatly improved to a certain extent.

The second experiment is to verify the control performance of the 6-DOF motion of the end-effector of the CDRPR. The tracking control is carried out on three translational motions (swaying, surging, and heaving) and three rotation motions (pitching, rolling, and yawing). In the other five directions' tracking, the heaving direction will move up to raise the end-effector. The control path, the cable length measured by the cable-length sensor, and the control tension are shown in Figures 9-14.

First, a trajectory in a swaying direction is considered. The control parameter is $\Delta=5, K=\operatorname{diag}\left(\begin{array}{llllll}2 & 2 & 2 & 2 & 2 & 2\end{array}\right)^{T}, C_{e}=$ $\operatorname{diag}\left(\begin{array}{llllll}40 & 15 & 20 & 15 & 15 & 15\end{array}\right)^{T}, Q=\operatorname{diag}\left(\begin{array}{llllll}8 & 8 & 8 & 8 & 8 & 8\end{array}\right)^{T}$, and the initial value of $\widehat{\mathbf{X}}_{P}^{B}$ is $\widehat{\mathbf{X}}_{p}^{B}=\left[\begin{array}{llllll}0 & 0 & -1.19 & 0 & 0 & 0\end{array}\right]^{T}$. The ideal signal is the red line shown in Figure 11(a); 

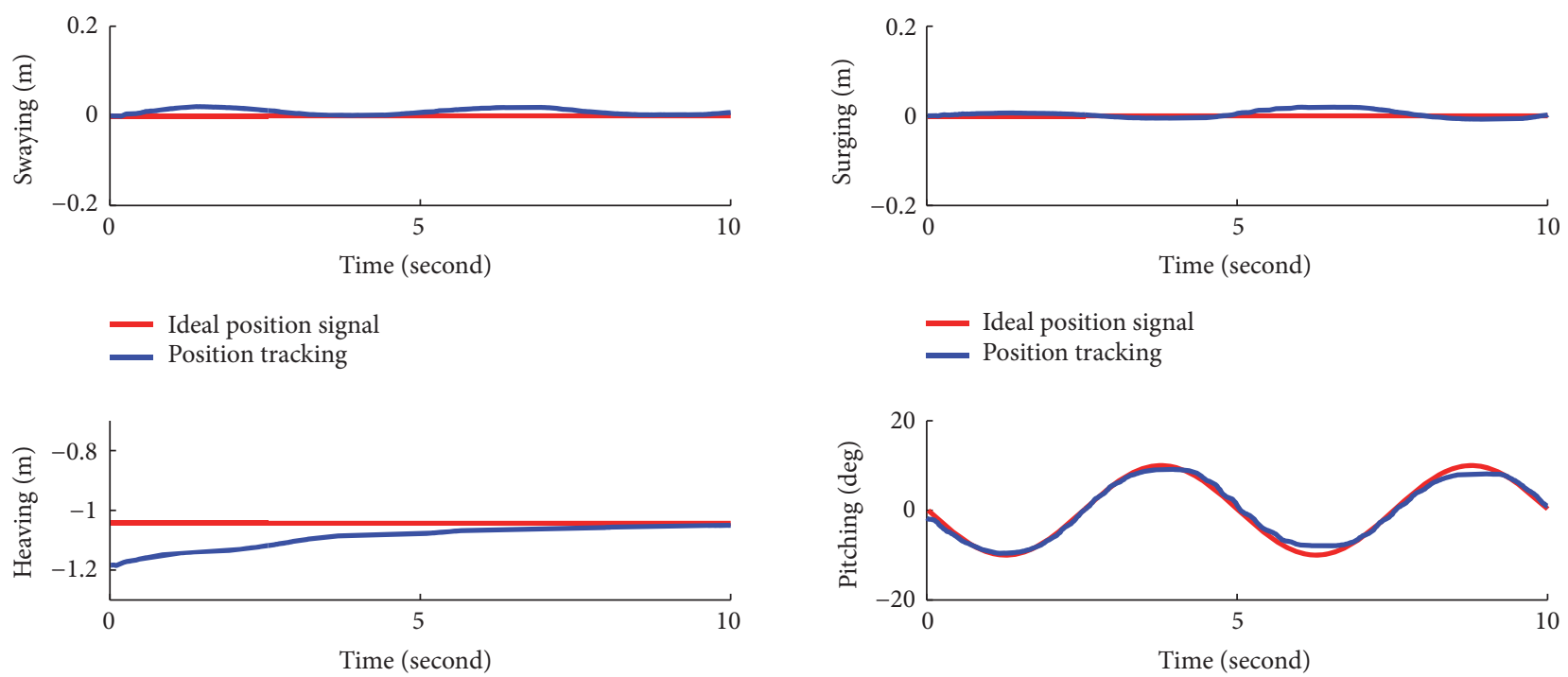

Ideal position signal Position tracking
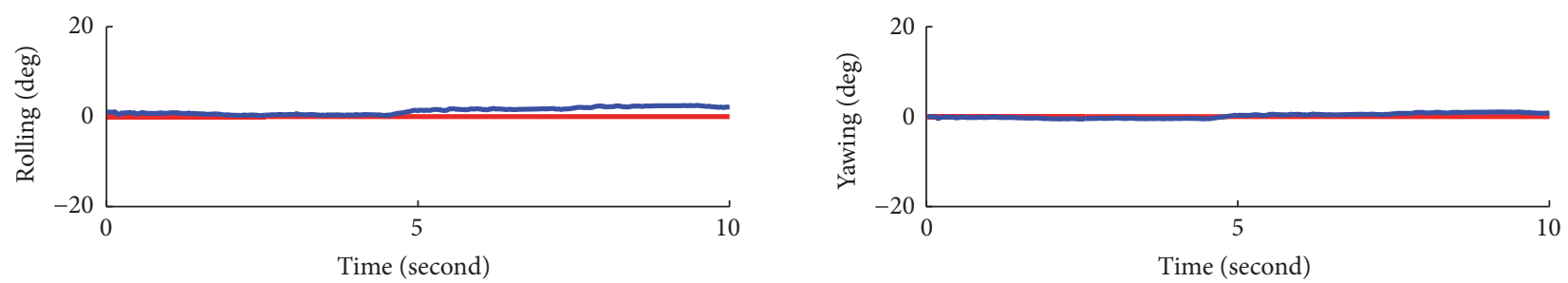

Ideal position signal Position tracking

(a)
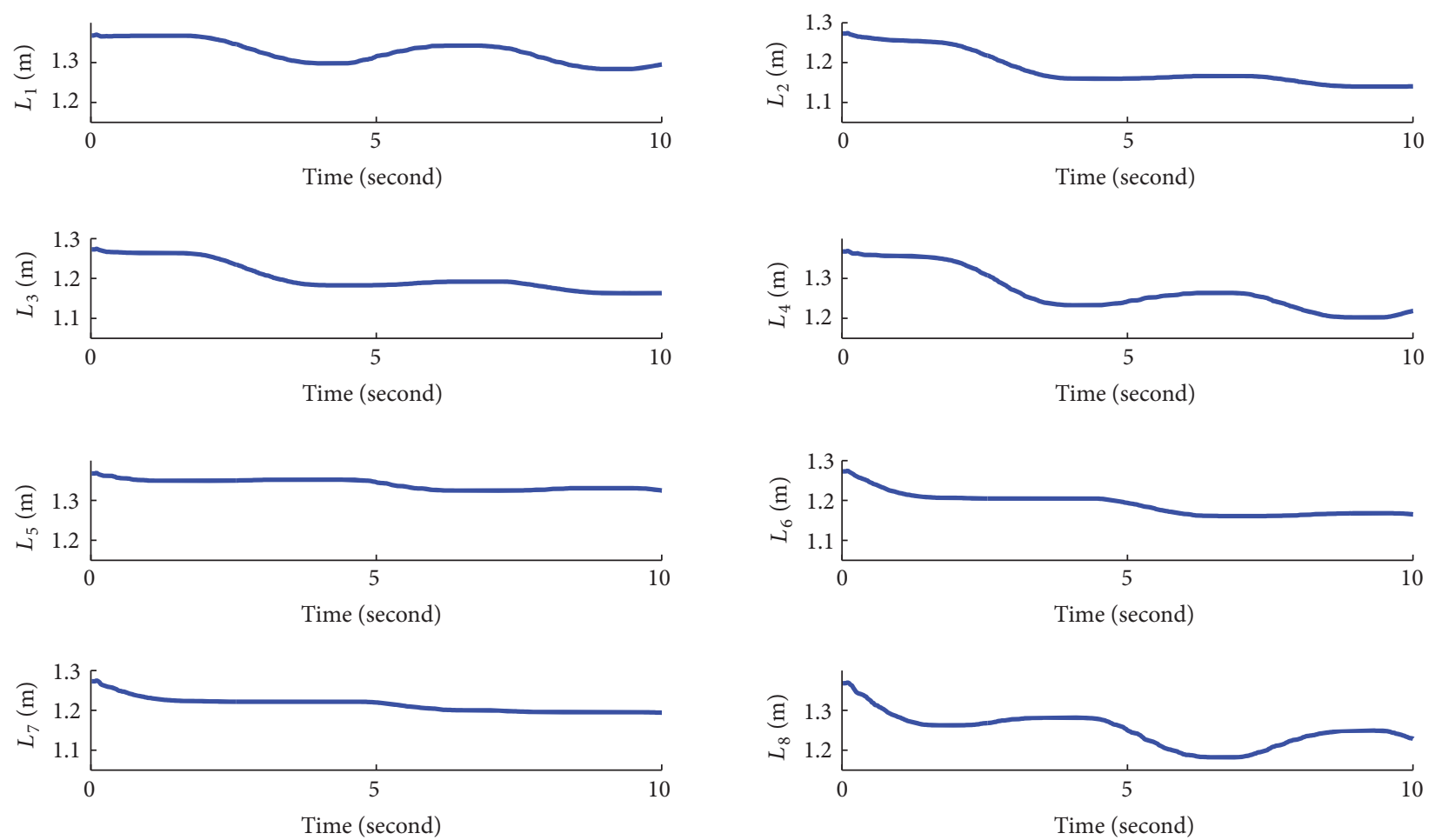

(b)

FIgURE 14: Continued. 

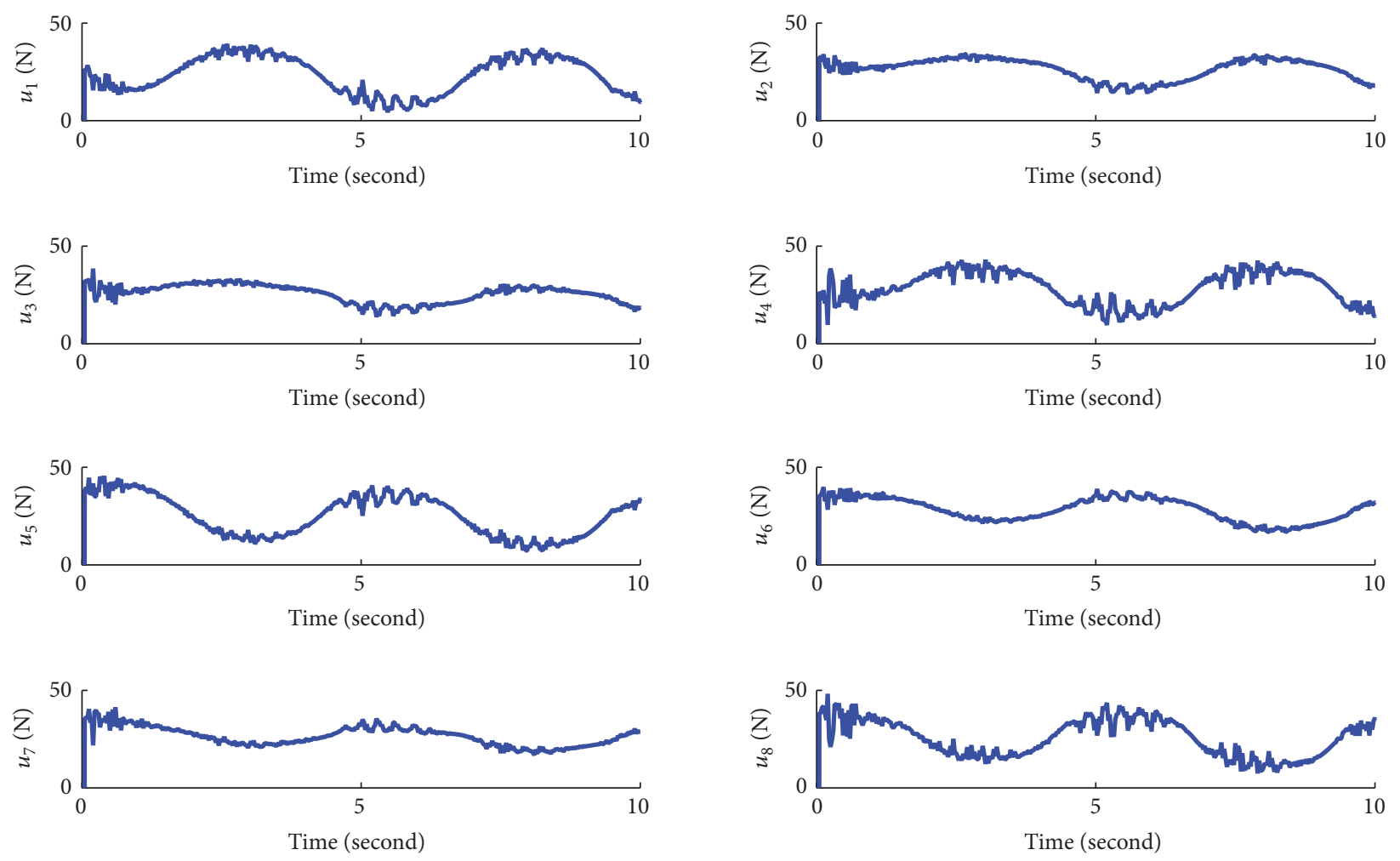

(c)

FIgURE 14: Tracking in a pitching direction.

the blue line is the position tracking path, which is the effector pose estimated by the cable-length sensor. The cable length is shown in Figure 11(b), and the control tension is shown in Figure 11(c).

Second, a trajectory in a surging direction is considered. The control parameter is $K=$ $\operatorname{diag}\left(\begin{array}{llllll}2 & 2 & 2 & 2 & 2 & 2\end{array}\right)^{T}, C_{e}=\operatorname{diag}\left(\begin{array}{llllll}15 & 25 & 20 & 15 & 15 & 15\end{array}\right)^{T}$, $Q=\operatorname{diag}\left(\begin{array}{llllll}12 & 12 & 12 & 12 & 12 & 12\end{array}\right)^{T}, \Delta=5$, and the initial value of $\widehat{\mathbf{X}}_{P}^{B}$ is $\widehat{\mathbf{X}}_{P}^{B}=\left[\begin{array}{llllll}0 & 0 & -1.19 & 0 & 0 & 0\end{array}\right]^{T}$. The ideal signal and tracking path are shown in Figure 12(a); the cable length is shown in Figure 12(b); and the control force is shown in Figure 12(c).

Third, a trajectory in a heaving direction is considered. The control parameter is $K=\operatorname{diag}\left(\begin{array}{llllll}2 & 2 & 2 & 2 & 2 & 2\end{array}\right)^{T}, C_{e}=$ $\operatorname{diag}\left(\begin{array}{llllll}15 & 15 & 55 & 15 & 15 & 15\end{array}\right)^{T}, Q=\operatorname{diag}\left(\begin{array}{llllll}7 & 7 & 7 & 7 & 7 & 7\end{array}\right)^{T}$, $\Delta=5$, and the initial value of $\widehat{\mathbf{X}}_{P}^{B}$ is $\widehat{\mathbf{X}}_{P}^{B}=$ $\left[\begin{array}{llllll}0 & 0 & -1.19 & 0 & 0 & 0\end{array}\right]^{T}$. The ideal signal and tracking path is shown in Figure 13(a); the cable length is shown in Figure 13(b); and the control force is shown in Figure 13(c).

Fourth, a trajectory in a pitching direction is considered. The control parameter is $K=$ $\operatorname{diag}\left(\begin{array}{llllll}2 & 2 & 2 & 2 & 2 & 2\end{array}\right)^{T}, C_{e}=\operatorname{diag}\left(\begin{array}{llllll}15 & 15 & 20 & 80 & 15 & 15\end{array}\right)^{T}$, $Q=\operatorname{diag}\left(\begin{array}{llllll}12 & 12 & 12 & 12 & 12 & 12\end{array}\right)^{T}, \Delta=5$, and the initial value of $\widehat{\mathbf{X}}_{P}^{B}$ is $\widehat{\mathbf{X}}_{P}^{B}=\left[\begin{array}{llllll}0 & 0 & -1.19 & 0 & 0 & 0\end{array}\right]^{T}$. The ideal signal and tracking path are shown in Figure 14(a); the cable length is shown in Figure 14(b); and the control force is shown in Figure 14(c).

Fifth, a trajectory in a rolling direction is considered. The control parameter is $K=\operatorname{diag}\left(\begin{array}{llllll}2 & 2 & 2 & 2 & 2 & 2\end{array}\right)^{T}$, $C_{e}=\operatorname{diag}\left(\begin{array}{llllll}15 & 25 & 20 & 15 & 50 & 15\end{array}\right)^{T}, \quad Q \quad=$ $\operatorname{diag}\left(\begin{array}{llllll}13 & 13 & 13 & 13 & 13 & 13\end{array}\right)^{T}, \Delta=5$, and the initial of $\widehat{\mathbf{X}}_{P}^{B}$ is $\widehat{\mathbf{X}}_{P}^{B}=\left[\begin{array}{llllll}0 & 0 & -1.19 & 0 & 0 & 0\end{array}\right]^{T}$. The ideal signal and tracking path are shown in Figure 15(a); the cable length is shown in Figure 15(b); and the control force is shown in Figure 15(c).

Sixth, a trajectory in a yawing direction is considered. The control parameter is $K=\operatorname{diag}\left(\begin{array}{llllll}2 & 2 & 2 & 2 & 2 & 2\end{array}\right)^{T}$, $C_{e}=\operatorname{diag}\left(\begin{array}{llllll}15 & 15 & 20 & 15 & 15 & 45\end{array}\right)^{T}, \quad Q \quad=$ $\operatorname{diag}\left(\begin{array}{llllll}12 & 12 & 12 & 12 & 12 & 12\end{array}\right)^{T}, \Delta=5$, and the initial value of $\widehat{\mathbf{X}}_{P}^{B}$ is $\widehat{\mathbf{X}}_{P}^{B}=\left[\begin{array}{llllll}0 & 0 & -1.19 & 0 & 0 & 0\end{array}\right]^{T}$. The ideal signal and tracking path are shown in Figure 16(a); the cable length is shown in Figure 16(b); and the control force is shown in Figure 16(c).

In Figures 11-16, (a) shows the experiment results of tracking in swaying, surging, heaving, pitching, rolling, and yawing directions based on the sliding mode controller. The ideal paths are drawn in red, while the actual paths are drawn in blue. It can be seen that the cable-length sensor can be used to obtain the current end-effector pose in real-time, and the system can track the end-effector motion of the end-effector 

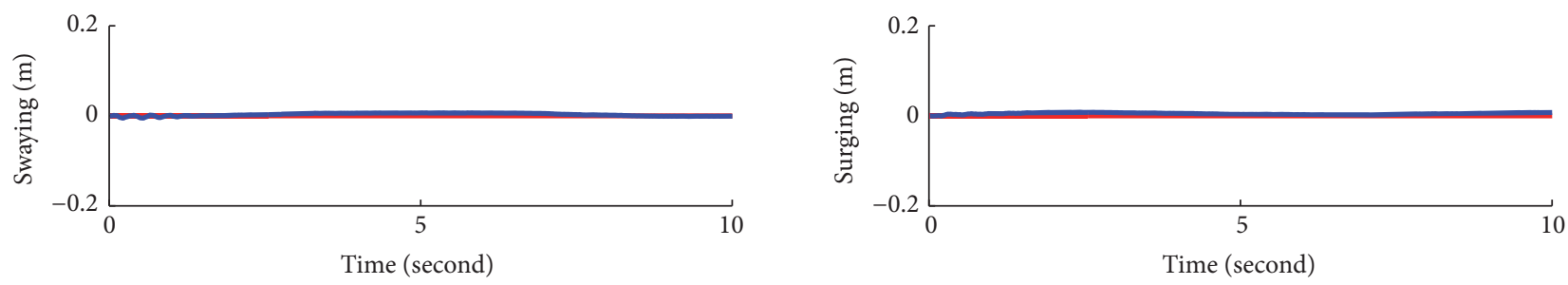

Ideal position signal

Position tracking
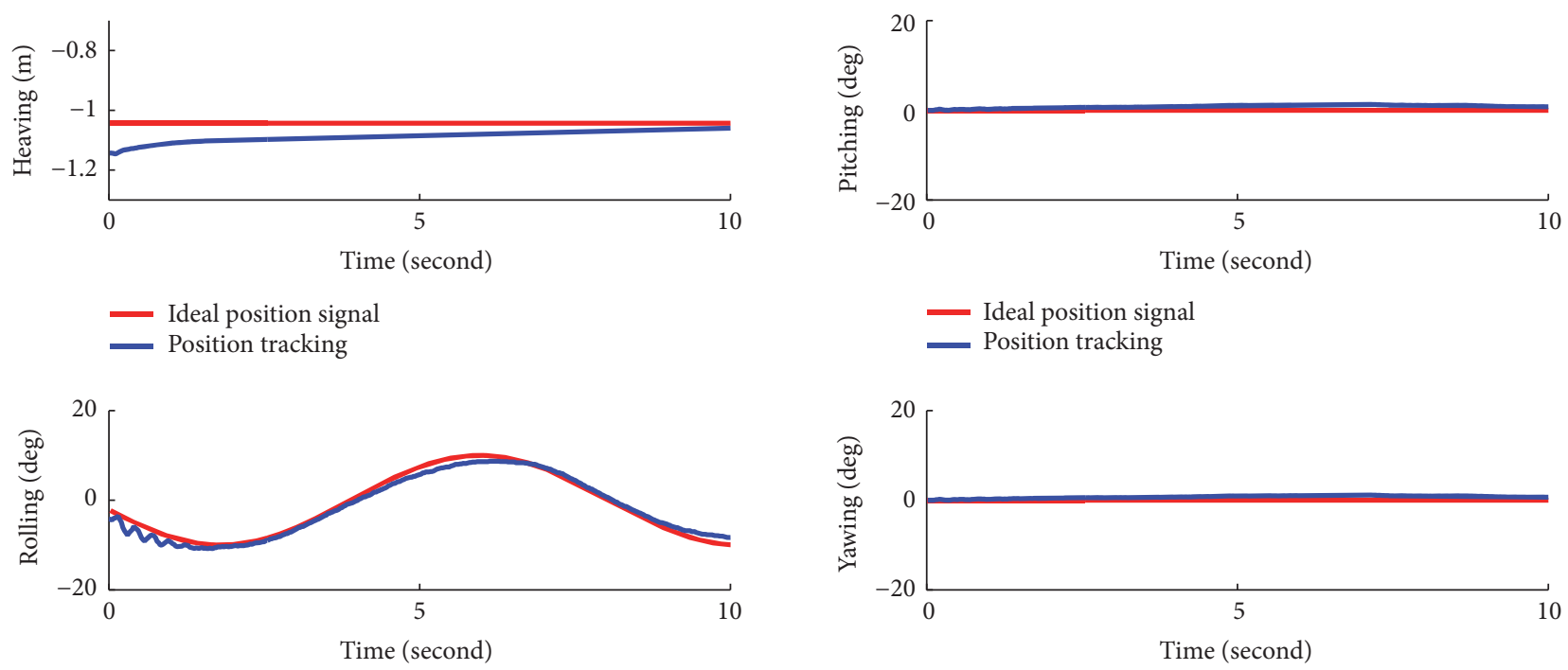

Ideal position signal

Position tracking

(a)
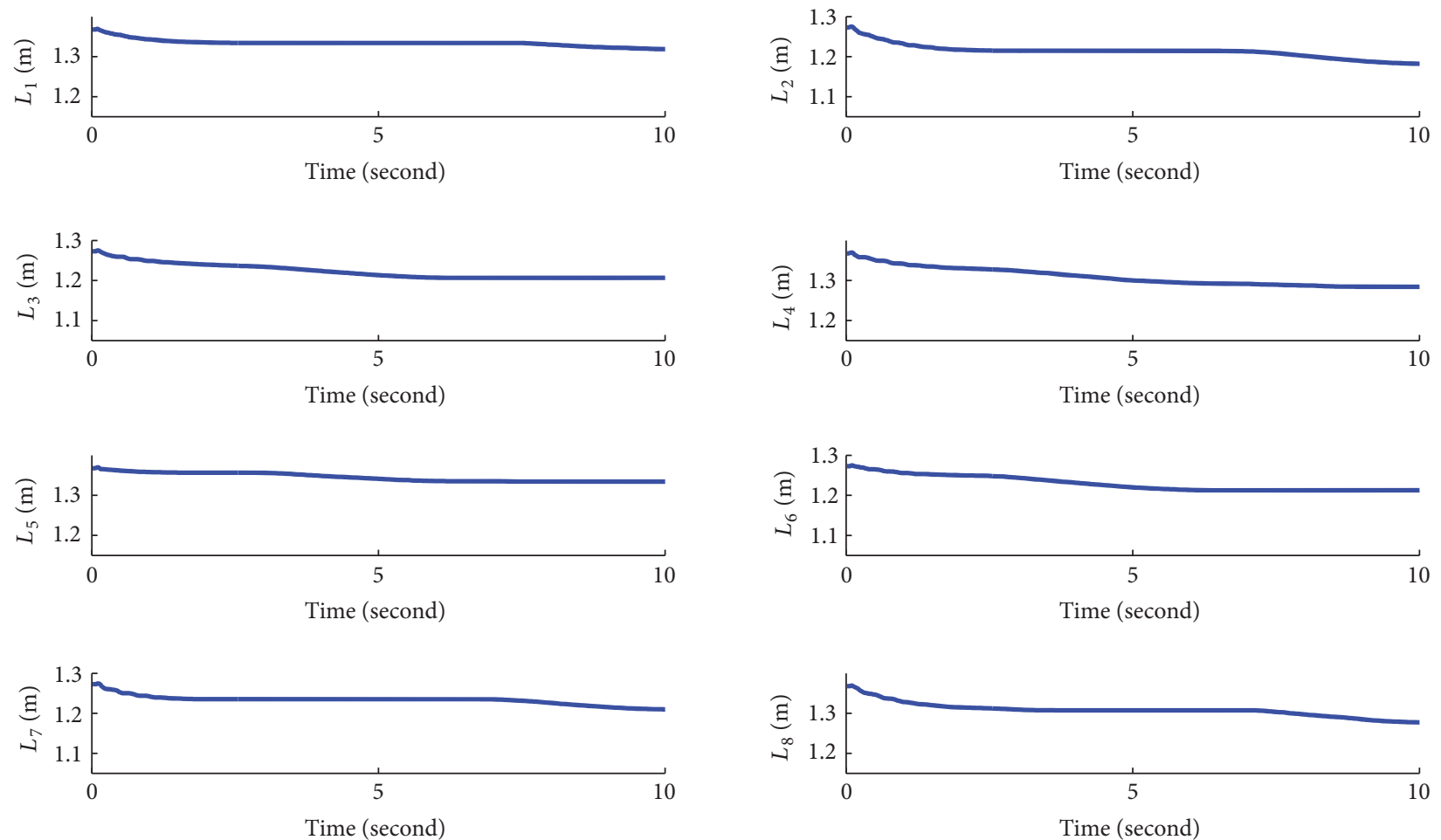

(b)

FIgURE 15: Continued. 

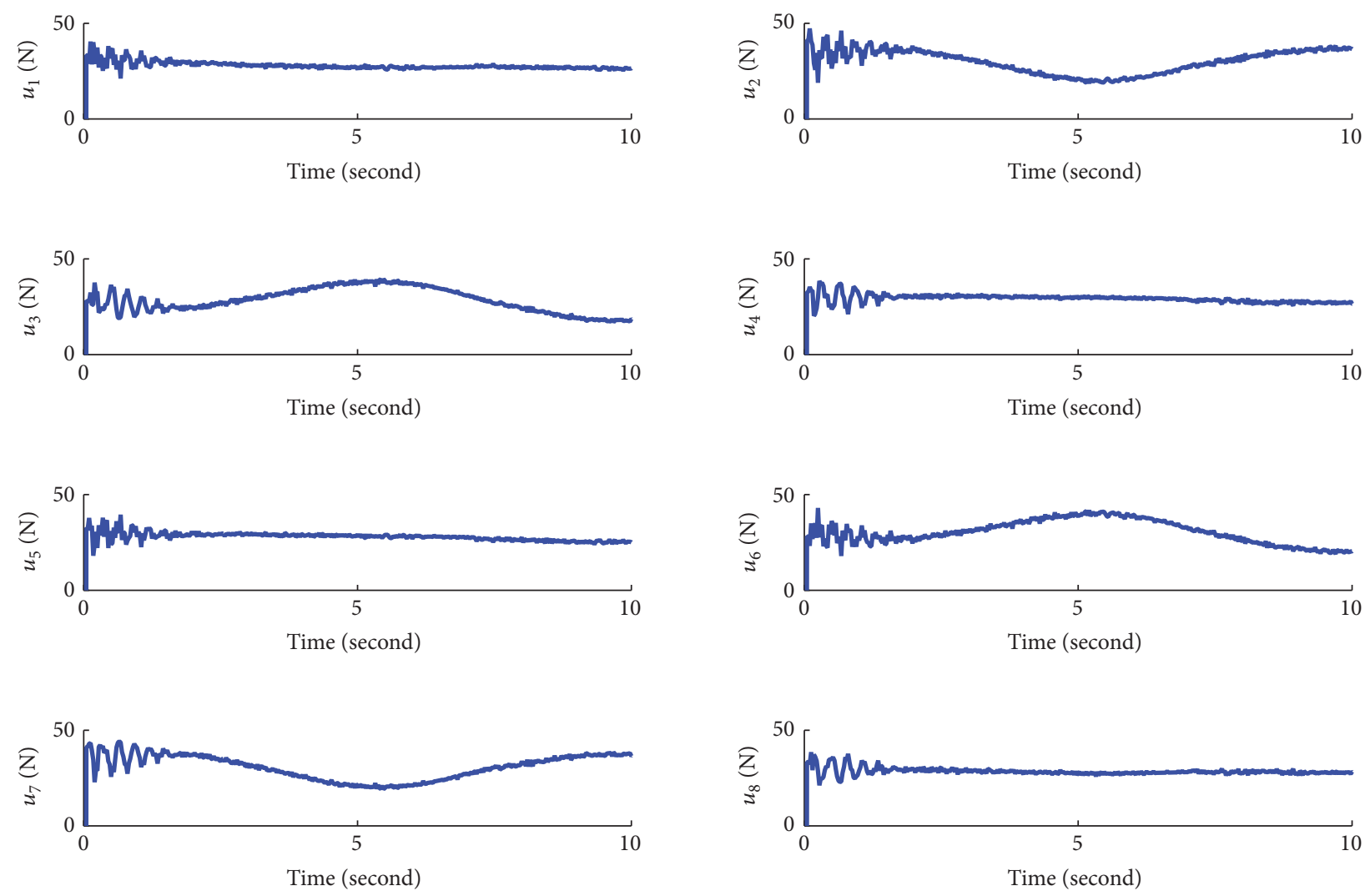

(c)

FIGURE 15: Tracking in a rolling direction.

in each direction accurately. When tracking a direction of movement, the other direction of movement is maintained at zero. However, the heaving direction of movement is not maintained at zero since the robot needs to raise the endeffector in the process of controlling.

In Figures 11-16, (b) shows the change in cable length measured by the cable-length sensor during the process of controlling. The cable length varies periodically with the periodic movement of the end-effector. In Figures 11-16, (c) shows the control tension of each rope, and there is no negative tension.

\section{Conclusions}

In this paper, the sliding mode control of the CDRPR with 6-DOF is studied based on cable-length sensor feedback. Under the control scheme of task space coordinates, the cable length measured by the cable-length sensor is used to solve the forward kinematics of the CDRPR in realtime; this is used as the feedback for the control system. The method used to solve the forward kinematics of the CDRPR is proposed based on the Tetrahedron Approach and Levenberg-Marquardt method. The high-dimensional nonlinear equations of mutual coupling were transformed into independent low-dimensional nonlinear equations using the tetrahedron approach, which is to ensure that the forward kinematics problem can be solved in real-time. Then, an iterative initial value estimation method for the LM method is proposed to ensure that the system can converge to the right point in the finite step.

The sliding mode control method based on exponential approach law is used to control the end-effector motion, and the influence of the sliding mode parameters on the control performance is simulated. Reaching speed parameter Q mainly affects the dynamic process of the switching function. The parameter can change the reaching speed from the system to the sliding surface. It can improve the dynamic quality of the system. The larger the $\mathbf{Q}$ is, the faster the system reaches the sliding surface. The sliding surface parameter $\mathbf{C}_{e}$ is to ensure that the sliding mode process is asymptotically stable and has a faster dynamic response speed. The larger the $\mathbf{C}_{e}$ is, the faster the response of the sliding mode process is. Therefore, the increase of $\mathbf{Q}$ and $\mathbf{C}_{e}$ can improve the system's rapidity. However, the parameter is too large, leading to the output of the control being too large, which often causes shaking in the actual control. Finally, the experimental results of 6-DOF position tracking on the principle prototype show that the robot can accurately track the desired position in six directions. Accuracy meets the system requirements. However, the tracking error is large in the wave trough; the influence of nonlinear factors such as friction may play a role, which may be addressed in future research. 

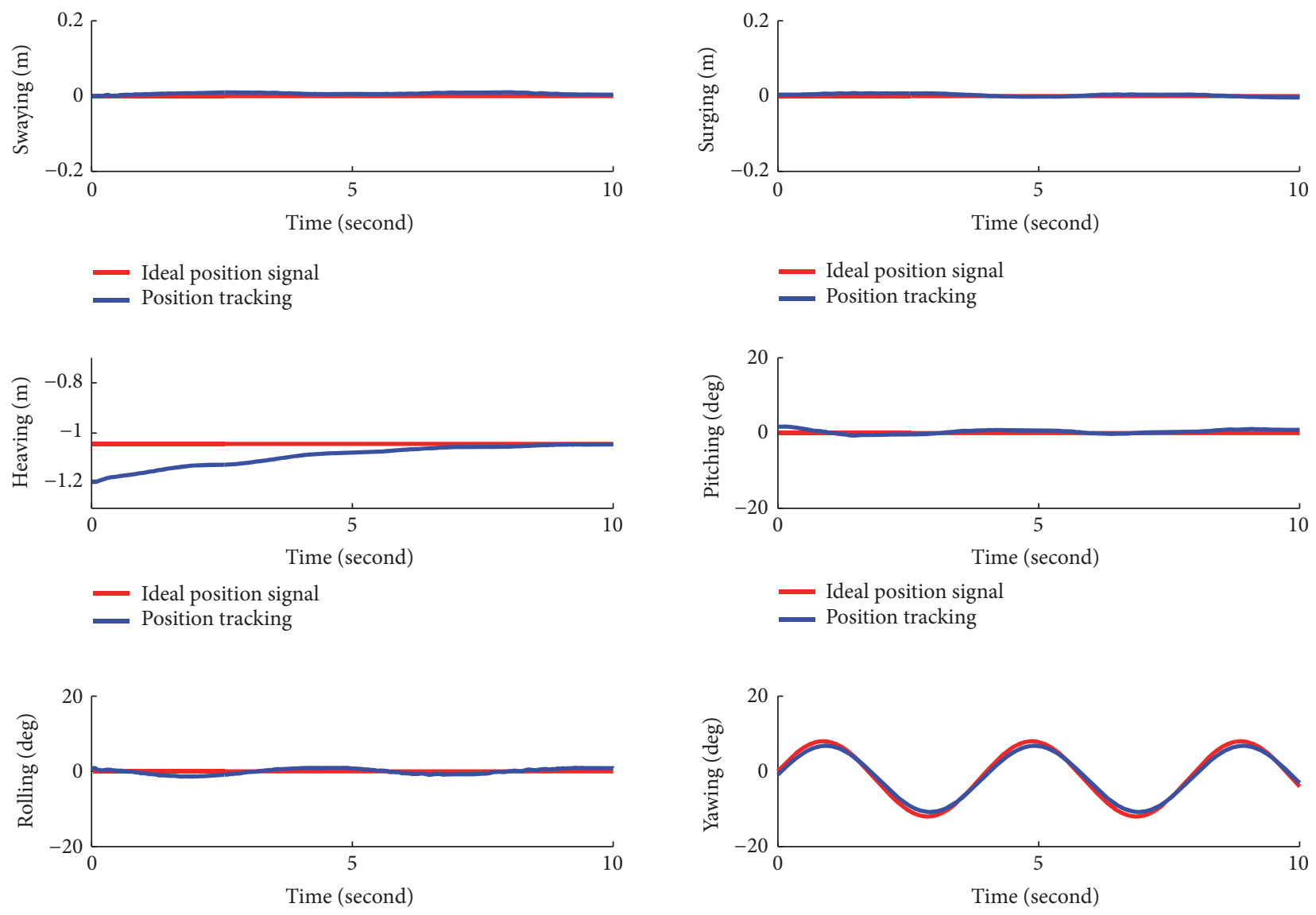

- Ideal position signal

_ Ideal position signal

_ Position tracking

(a)
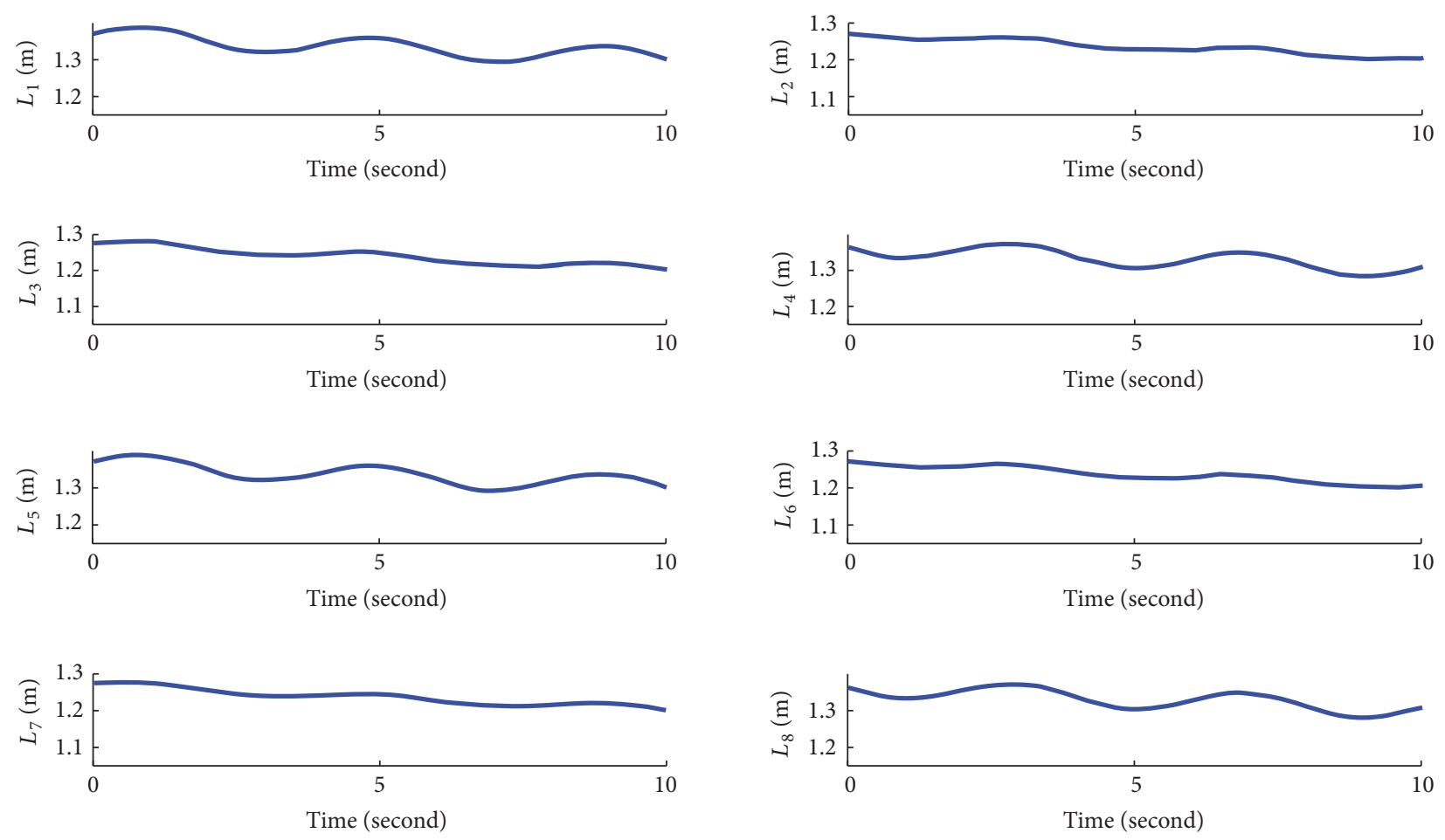

(b)

Figure 16: Continued. 

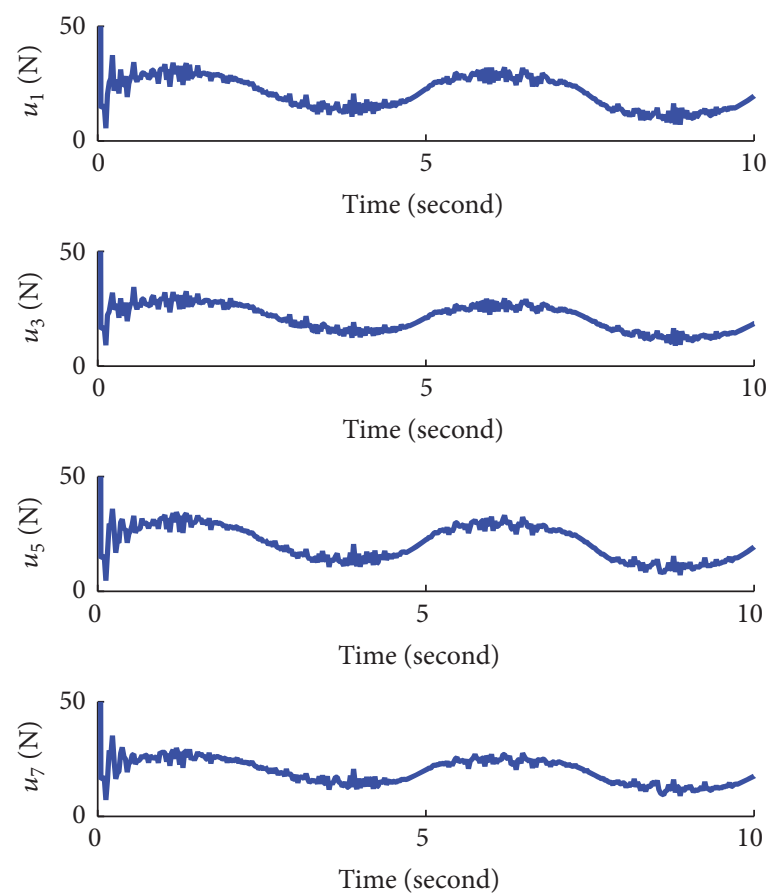
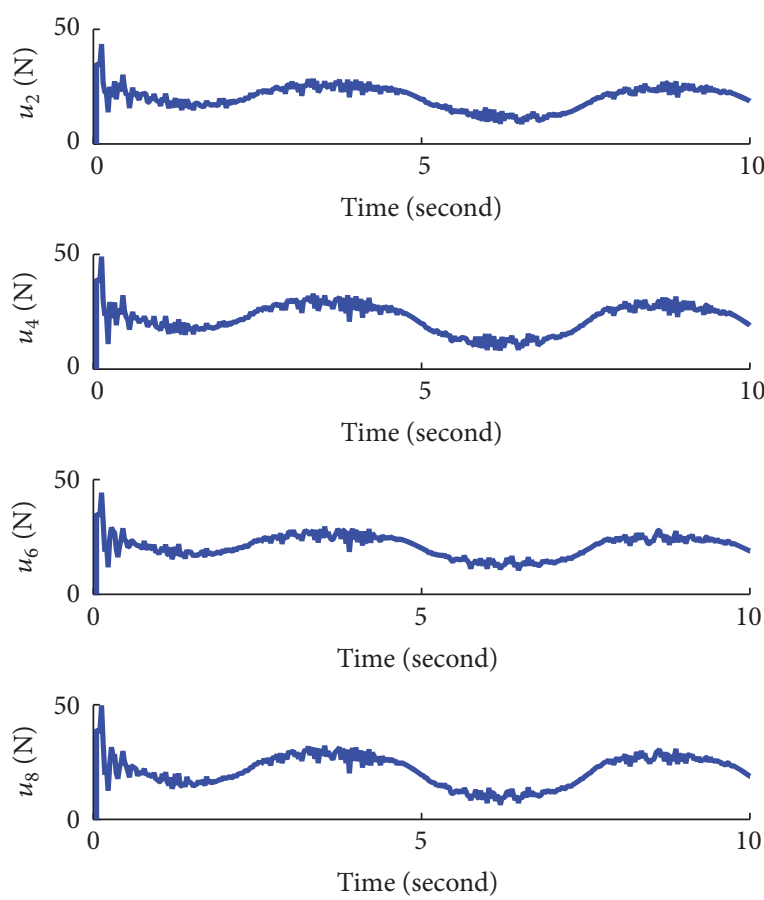

(c)

FIGURE 16: Tracking in a yawing direction.

\section{Conflicts of Interest}

The authors declare no conflicts of interest.

\section{Acknowledgments}

The authors acknowledge the financial support that was given under the Project supported by the National Ministries and Commissions of China (Grant no. 4010406020204).

\section{References}

[1] S. Oh and S. Agrawal, "Cable suspended planar robots with redundant cables: controllers with positive tensions," IEEE/ Transactions on Robotics, vol. 21, no. 3, pp. 457-465, 2005.

[2] R. Babaghasabha, M. A. Khosravi, and H. D. Taghirad, "Adaptive robust control of fully-constrained cable driven parallel robots," Mechatronics, vol. 25, pp. 27-36, 2015.

[3] S. Fang, D. Franitza, M. Torlo, F. Bekes, and M. Hiller, "Motion control of a tendon-based parallel manipulator using optimal tension distribution," IEEE/ASME Transactions on Mechatronics, vol. 9, no. 3, pp. 561-568, 2004.

[4] P. Gholami, M. M. Aref, and H. D. Taghirad, "On the control of the KNTU CDRPM: a cable driven redundant parallel manipulator," in Proceedings of the IEEE/RSJ International Conference on Intelligent Robots and Systems (IROS '08), pp. 2404-2409, Nice, France, September 2008.

[5] B. Zi, B. Y. Duan, J. L. Du, and H. Bao, "Dynamic modeling and active control of a cable-suspended parallel robot," Mechatronics, vol. 18, no. 1, pp. 1-12, 2008.

[6] M. H. Korayem, H. Tourajizadeh, M. Jalali, and E. Omidi, "Optimal path planning of spatial cable robot using optimal sliding mode control," International Journal of Advanced Robotic Systems, vol. 9, 2012.

[7] S.-R. Oh, J.-C. Ryu, and S. K. Agrawal, "Dynamics and control of a helicopter carrying a payload using a cable-suspended robot," Journal of Mechanical Design, Transactions of the ASME, vol.128, no. 5, pp. 1113-1121, 2006.

[8] Y. Hu, L. Tao, J. Jia, and W. Lv, "Control and simulation of cable-driven parallel robots in offshore cargo handling," in Proceedings of the 11th World Congress on Intelligent Control and Automation, WCICA 2014, pp. 2451-2455, Shenyang, China, July 2014.

[9] A. Vafaei, M. M. Aref, and H. D. Taghirad, "Integrated controller for an over-constrained cable driven parallel manipulator: KNTU CDRPM," in Proceedings of the IEEE International Conference on Robotics and Automation (ICRA '10), pp. 650-655, Anchorage, Alaska, USA, May 2010.

[10] F. Paccot, N. Andreff, and P. Martinet, "A review on the dynamic control of parallel kinematic machines: theory and experiments," International Journal of Robotics Research, vol. 28, no. 3, pp. 395-416, 2009.

[11] H. Bayani, M. T. Masouleh, and A. Kalhor, "An experimental study on the vision-based control and identification of planar cable-driven parallel robots," Robotics and Autonomous Systems, vol. 75, pp. 187-202, 2016.

[12] T. Dallej, M. Gouttefarde, N. Andreff, M. Michelin, and P. Martinet, "Towards vision-based control of cable-driven parallel robots," in Proceedings of the IEEE/RSJ International Conference on Intelligent Robots and Systems: Celebrating 50 Years of Robotics (IROS '11), pp. 2855-2860, San Francisco, Calif, USA, September 2011.

[13] T. Dallej, M. Gouttefarde, N. Andreff, R. Dahmouche, and P. Martinet, "Vision-based modeling and control of largedimension cable-driven parallel robots," in Proceedings of 25th 
IEEE/RSJ International Conference on Robotics and Intelligent Systems, IROS 2012, pp. 1581-1586, Algarve, Portugal, October 2012.

[14] L. Yingjie, Z. Wenbai, and R. Gexue, "Feedback control of a cable-driven gough-stewart platform," IEEE Transactions on Robotics, vol. 22, pp. 198-202, 2006.

[15] R. Ramadour, F. Chaumette, and J.-P. Merlet, "Grasping objects with a cable-driven parallel robot designed for transfer operation by visual servoing," in Proceedings of 2014 IEEE International Conference on Robotics and Automation, ICRA 2014, pp. 4463-4468, chn, June 2014.

[16] R. Chellal, L. Cuvillon, and E. Laroche, "A kinematic visionbased position control of a 6-dof cable-driven parallel robot," Mechanisms and Machine Science, vol. 32, pp. 213-225, 2015.

[17] W. S. Newman, C. E. Birkhimer, R. J. Horning, and A. T. Wilkey, "Calibration of a Motoman P8 robot based on laser tracking," in Proceedings of the IEEE International Conference on Robotics and Automation, vol. 4, pp. 3597-3602, April 2000.

[18] A. M. Lytle, K. S. Saidi, R. V. Bostelman, W. C. Stone, and N. A. Scott, "Adapting a teleoperated device for autonomous control using three-dimensional positioning sensors: experiences with the NIST RoboCrane," Automation in Construction, vol. 13, no. 1, pp. 101-118, 2004.

[19] D.-H. Kim, J.-W. Lee, K.-T. Park, and J.-H. Oh, "Closed-form kinematic solution of a non-parallel cable reeving crane system," Proceedings of the Institution of Mechanical Engineers, Part C: Journal of Mechanical Engineering Science, vol. 217, no. 2, pp. 257-270, 2003.

[20] S.-H. Chen and L.-C. Fu, "The forward kinematics of the 6-6 Stewart platform using extra sensors," in Proceedings of 2006 IEEE International Conference on Systems, Man and Cybernetics, vol. 6, pp. 4671-4676, October 2006.

[21] J.-P. Merlet, "Closed-form resolution of the direct kinematics of parallel manipulators using extra sensors data," in Proceedings of the IEEE International Conference on Robotics and Automation, pp. 200-204, Singapore, Singapore, May 1993.

[22] V. Parenti-Castelli and R. Di Gregorio, "Closed-form solution of the direct kinematics of the 6-3 type Stewart Platform using one extra sensor," Meccanica, vol. 31, no. 6, pp. 705-714, 1996.

[23] P. Gallina, A. Rossi, and R. L. Williams, "Planar cable-directdriven robots. Part II: dynamics and control," in Proceedings of the 2001 ASME Design Technical Conferences 27th Design Automation Conference, Pittsburgh, Pa, USA, 2001.

[24] M. A. Khosravi and H. D. Taghirad, "Robust PID control of fully-constrained cable driven parallel robots," Mechatronics, vol. 24, no. 2, pp. 87-97, 2014.

[25] R. L. Williams, B. Snyder, J. S. Albus, and R. V. Bostelman, "Seven-DOF cable-suspended robot with independent metrology," in Proceedings of the ASME Design Engineering Technical Conferences and Computers and Information in Engineering Conference, pp. 1-9, Salt Lake City, Utah, USA, 2004.

[26] C.-F. Yang, S.-T. Zheng, J. Jin, S.-B. Zhu, and J.-W. Han, "Forward kinematics analysis of parallel manipulator using modified global Newton-Raphson method," Journal of Central South University of Technology (English Edition), vol. 17, no. 6, pp. 1264-1270, 2010.

[27] W. Sun-an and W. A. N. Ya-min, "A mixed real-time algorithm for the forward kinematics of stewart parallel manipulator," Journal of Electronic Science and Technology, pp. 173-180, 2006.

[28] A. Morell, L. Acosta, and J. Toledo, "An artificial intelligence approach to forward kinematics of Stewart Platforms," in
Proceedings of 2012 20th Mediterranean Conference on Control and Automation, MED 2012, pp. 433-438, Barcelona, Spain, July 2012.

[29] A. Morell, M. Tarokh, and L. Acosta, "Solving the forward kinematics problem in parallel robots using Support Vector Regression," Engineering Applications of Artificial Intelligence, vol. 26, no. 7, pp. 1698-1706, 2013.

[30] M. Tarokh, "Real time forward kinematics solutions for general Stewart platforms," in Proceedings of 2007 IEEE International Conference on Robotics and Automation, ICRA'07, pp. 901-906, Roma, Italy, April 2007.

[31] J. He, H. Gu, and Z. Wang, "Solving the forward kinematics problem of six-DOF Stewart platform using multi-task Gaussian process," Proceedings of the Institution of Mechanical Engineers, Part C: Journal of Mechanical Engineering Science, vol. 227, no. 1, pp. 161-169, 2013.

[32] T.-Y. Lee and J.-K. Shim, "Forward kinematics of the general 6-6 Stewart platform using algebraic elimination," Mechanism and Machine Theory, vol. 36, no. 9, pp. 1073-1085, 2001.

[33] T.-Y. Lee and J.-K. Shim, "Algebraic elimination-based real-time forward kinematics of the 6-6 stewart platform with planar base and platform," in Proceedings of the ICRA. IEEE International Conference on Robotics and Automation (Cat. No.01CH37164), vol. 2, pp. 1301-1306, 2001.

[34] Y. Xu and F. Xi, "A real-time method for solving the forward kinematics of a tripod with fixed-length legs," Journal of Manufacturing Science and Engineering, Transactions of the ASME, vol. 128, no. 1, pp. 204-212, 2006.

[35] A. Pott, "An algorithm for real-time forward kinematics of cable-driven parallel robots," in Advances in robot kinematics: Motion in man and machine: Motion in Man and Machine, J. Lenarcic, M. M. Stanisic, and M. M. Stanisic, Eds., pp. 529-538, Springer, Dordrecht, Netherlands, 2010.

[36] S.-K. Song and D.-S. Kwon, "A tetrahedron approach for a unique closed-form solution of the forward kinematics of sixdof parallel mechanisms with multiconnected joints," Journal of Robotic Systems, vol. 19, no. 6, pp. 269-281, 2002.

[37] Y. Hu, L. Tao, and W. Lv, "Anti-pendulation analysis of parallel wave compensation systems," Proceedings of the Institution of Mechanical Engineers Part M: Journal of Engineering for the Maritime Environment, vol. 230, no. 1, pp. 268-271, 2014. 


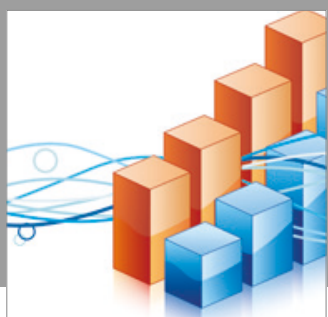

Advances in

Operations Research

vatersals

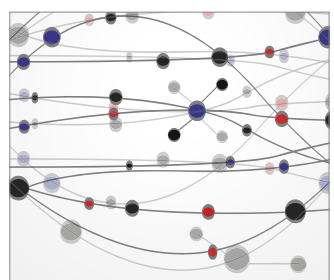

\section{The Scientific} World Journal
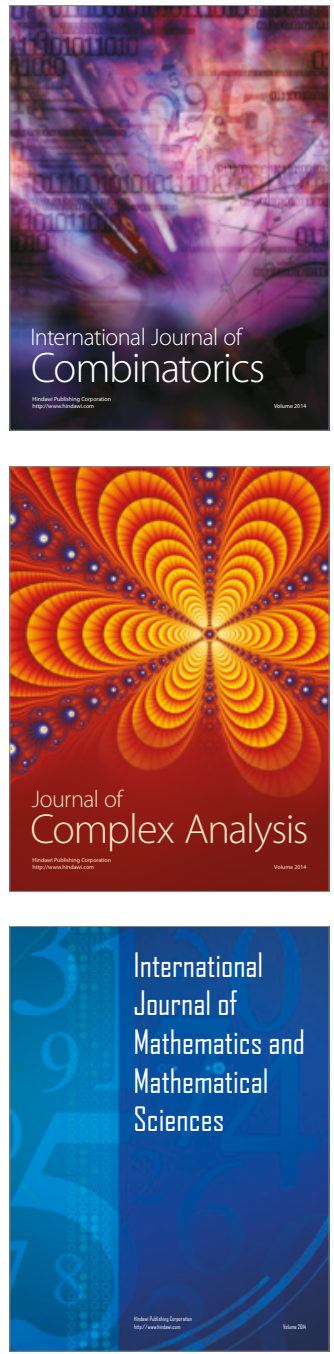
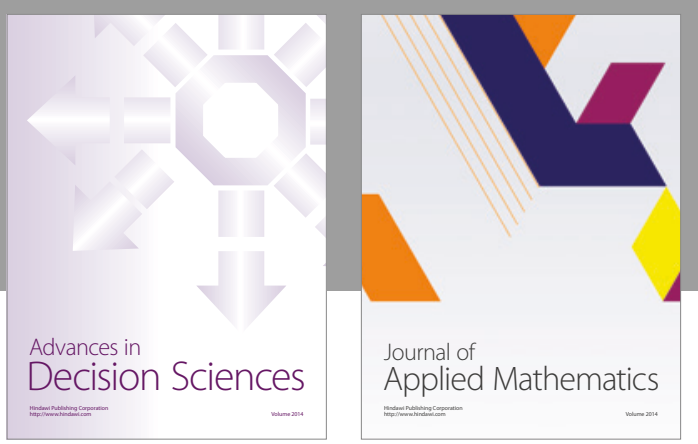

Algebra

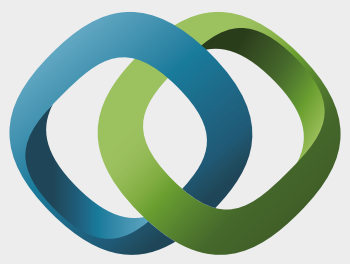

\section{Hindawi}

Submit your manuscripts at

https://www.hindawi.com
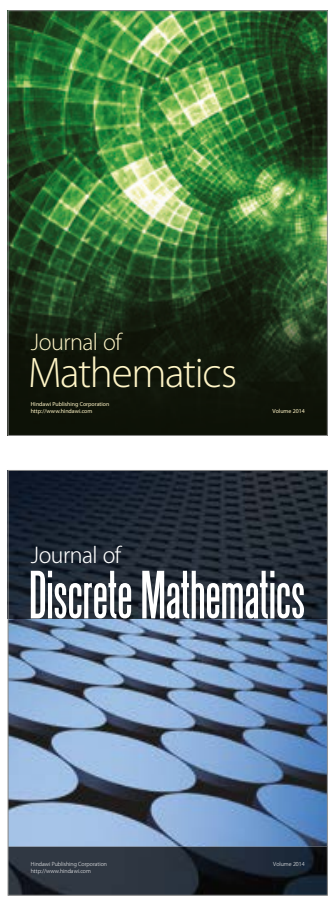

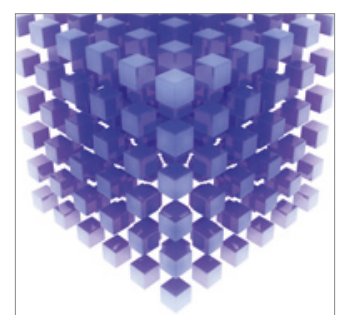

Mathematical Problems in Engineering
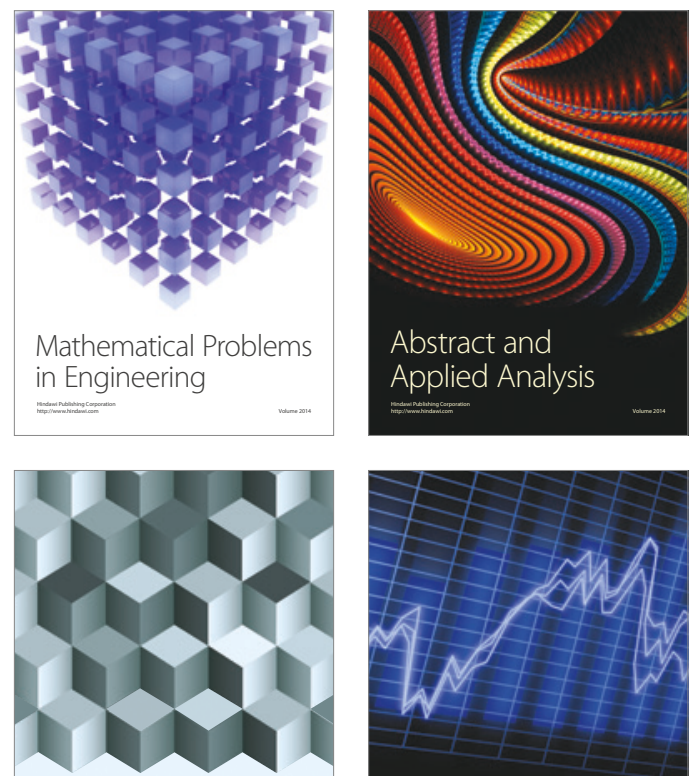

Journal of

Function Spaces

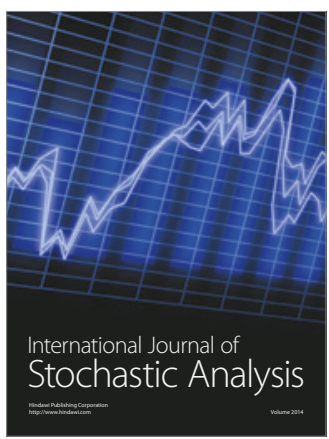

Probability and Statistics
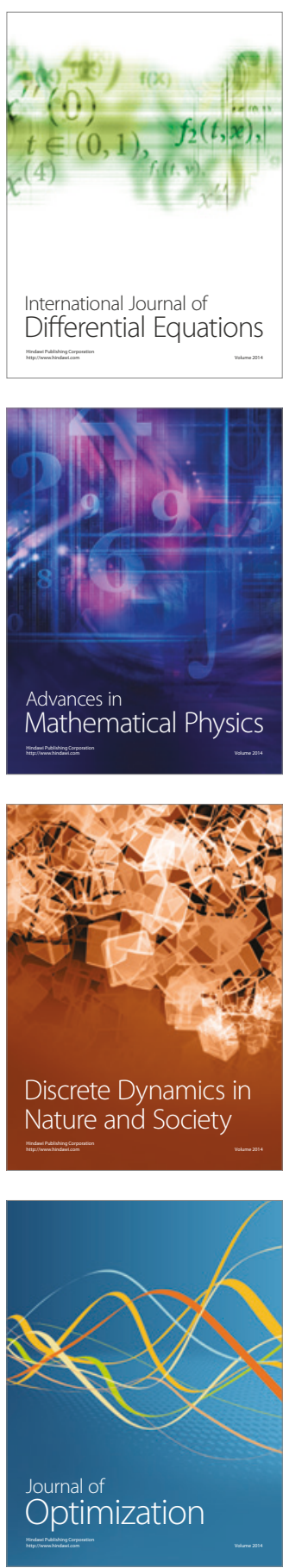